\title{
Polyfunctional Acrylic Non-Isocyanate Hydroxyurethanes as Photo-curable Thermosets for 3D Printing
}

\section{Supporting Information}

Vitalij Schimpf ${ }^{\dagger, \ddagger}$, Anne Asmacher ${ }^{\dagger}$, Bernd Bruchmann $^{\ddagger}$, Andre Fuchs $^{\ddagger, \S}$ and Rolf Mülhaupt ${ }^{\dagger, \ddagger} *$ †Freiburg Materials Research Center (FMF) and Institute for Macromolecular Chemistry, University of Freiburg, Stefan-Meier-Strasse 21 and 31, 79104 Freiburg, Germany ¥JONAS - Joint Research on Advanced Materials and Systems, Advanced Materials \& Systems Research, BASF SE, Carl-Bosch-Strasse 38, 67056 Ludwigshafen, Germany §BASF Schweiz AG, Mattenstrasse 22, 4057 Basel, Switzerland

\section{Table of Contents}

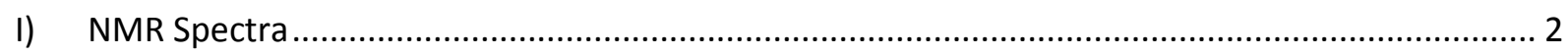

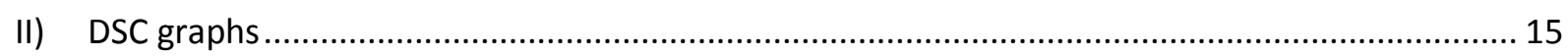

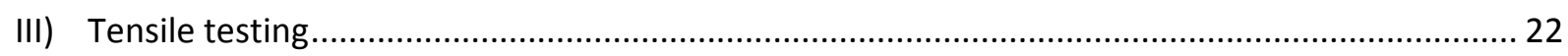

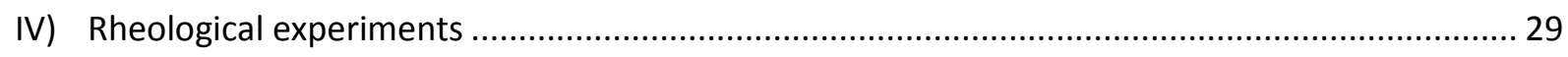

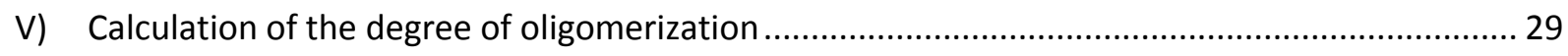

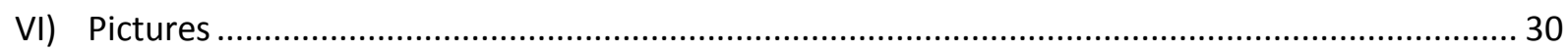




\section{I) NMR Spectra}
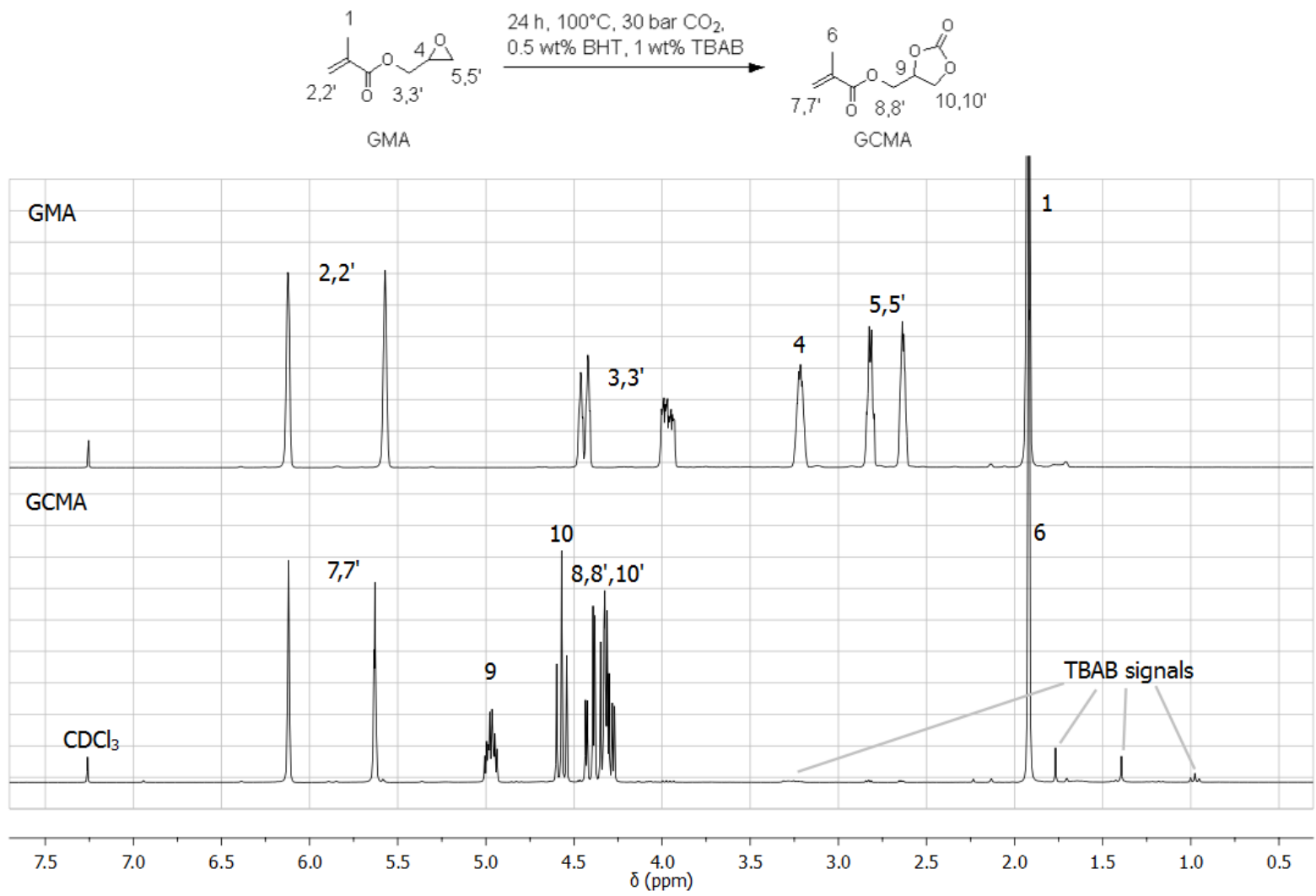

Figure S 1: ${ }^{1} \mathrm{H}-\mathrm{NMR}$ spectrum $\left(\mathrm{CDCl}_{3}\right)$ of glycidyl methacrylate (GMA) and glycerol carbonate dimethacrylate (GCMA).

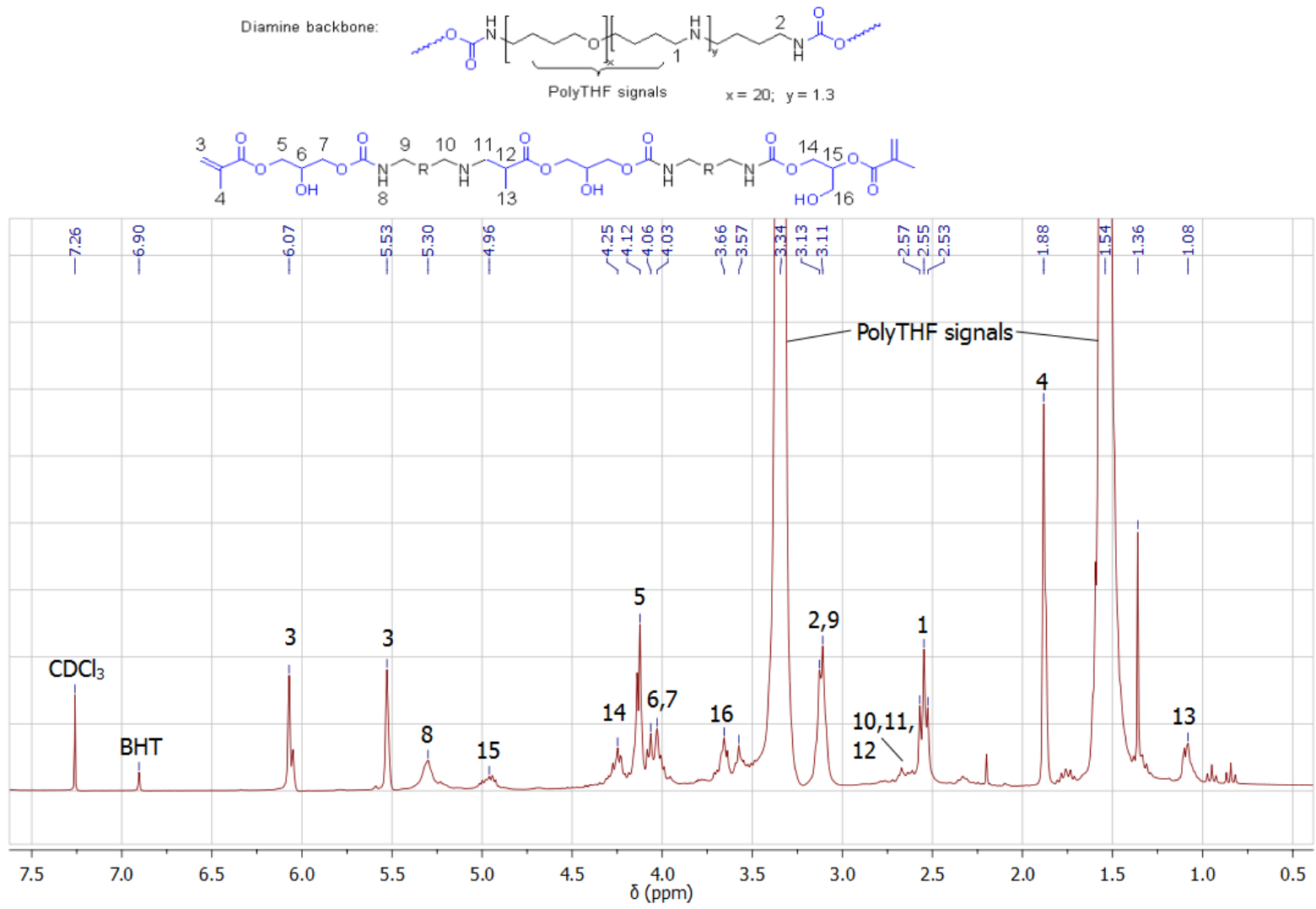

Figure S 2: ${ }^{1} \mathrm{H}-\mathrm{NMR}$ spectrum $\left(\mathrm{CDCl}_{3}\right)$ of PTHFA-G. 


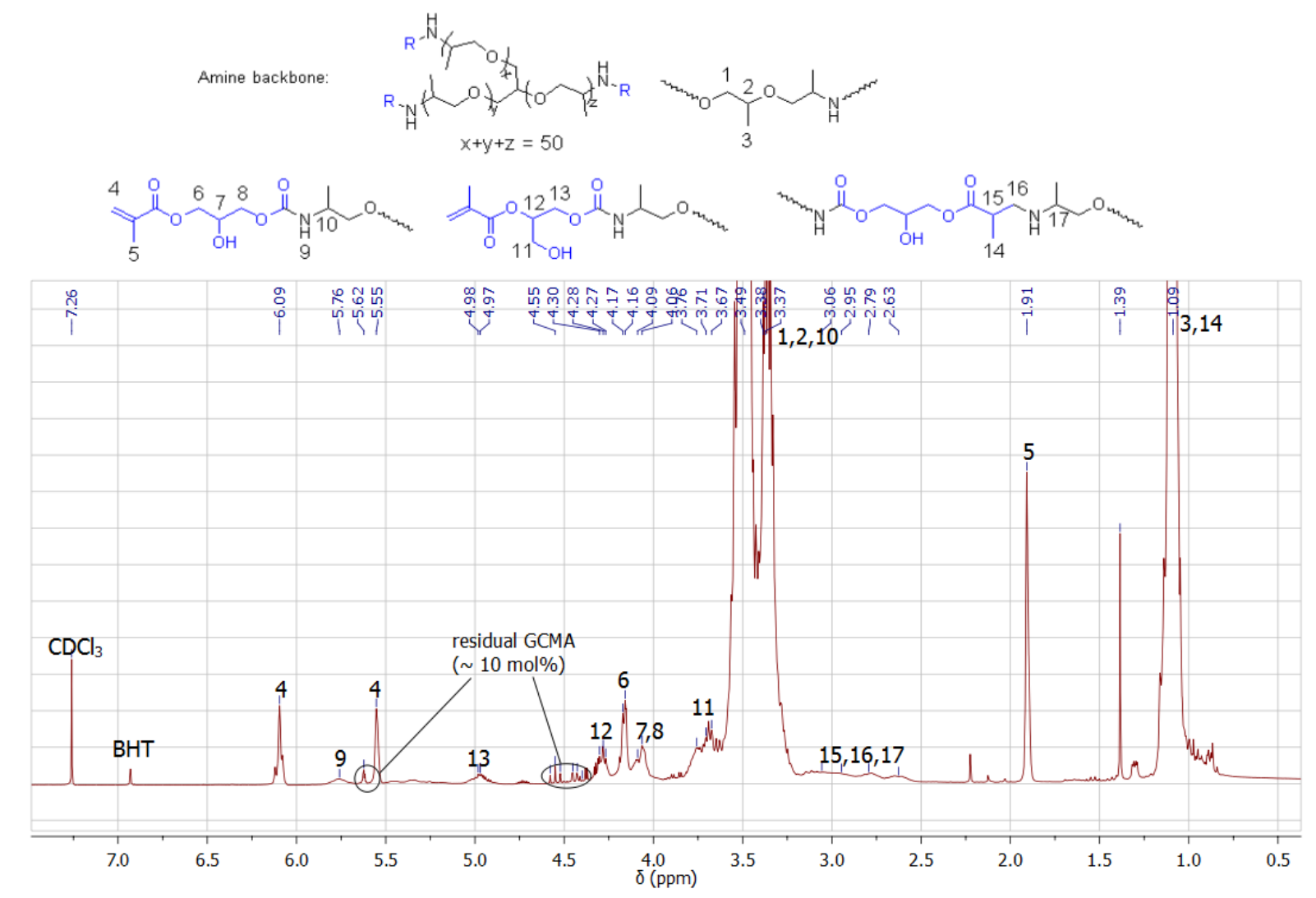

Figure S 3: ${ }^{1} \mathrm{H}-\mathrm{NMR}$ spectrum $\left(\mathrm{CDCl}_{3}\right)$ of JT3000-G.

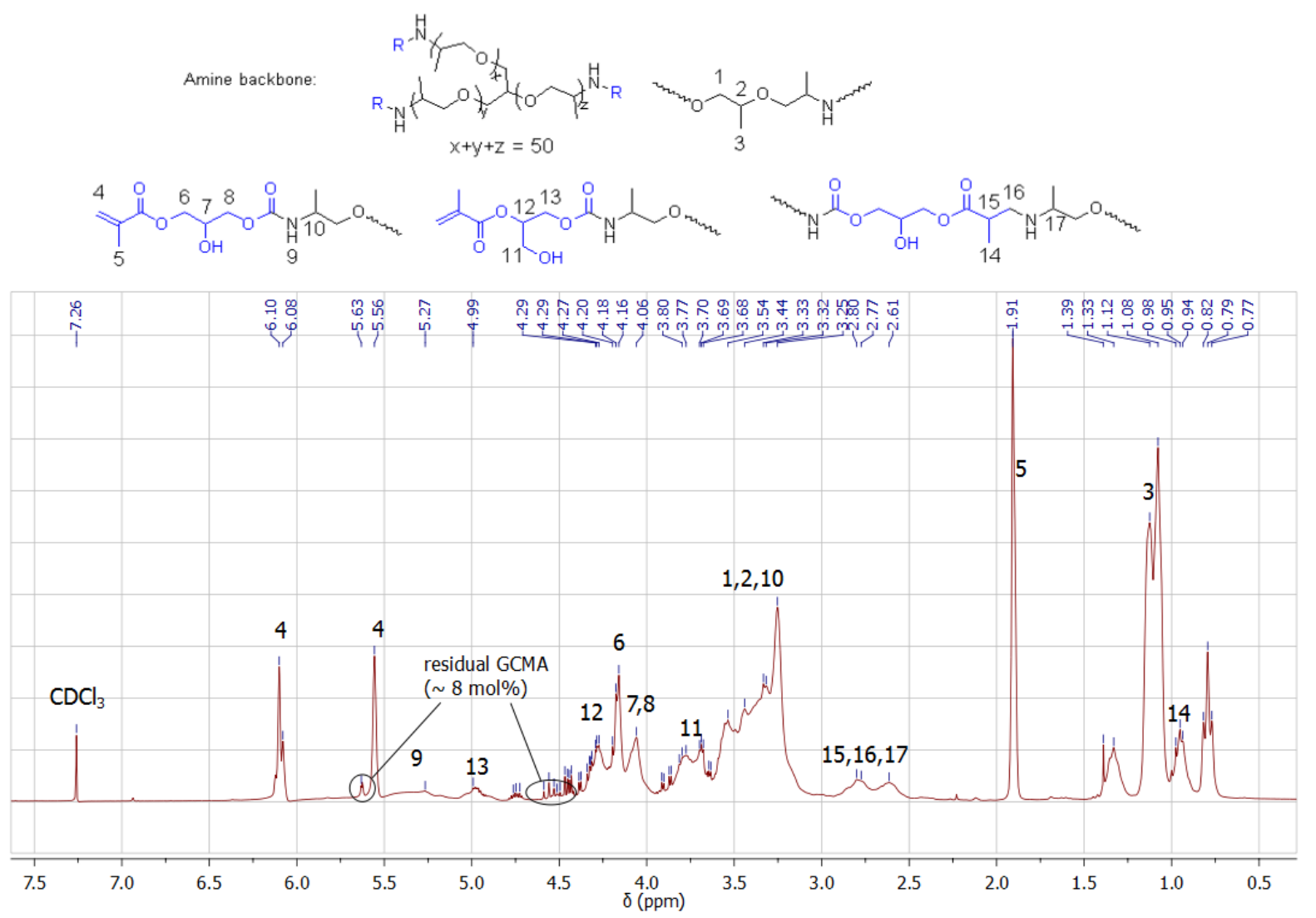

Figure S 4: ${ }^{1} \mathrm{H}$-NMR spectrum $\left(\mathrm{CDCl}_{3}\right)$ of JT403-G. 


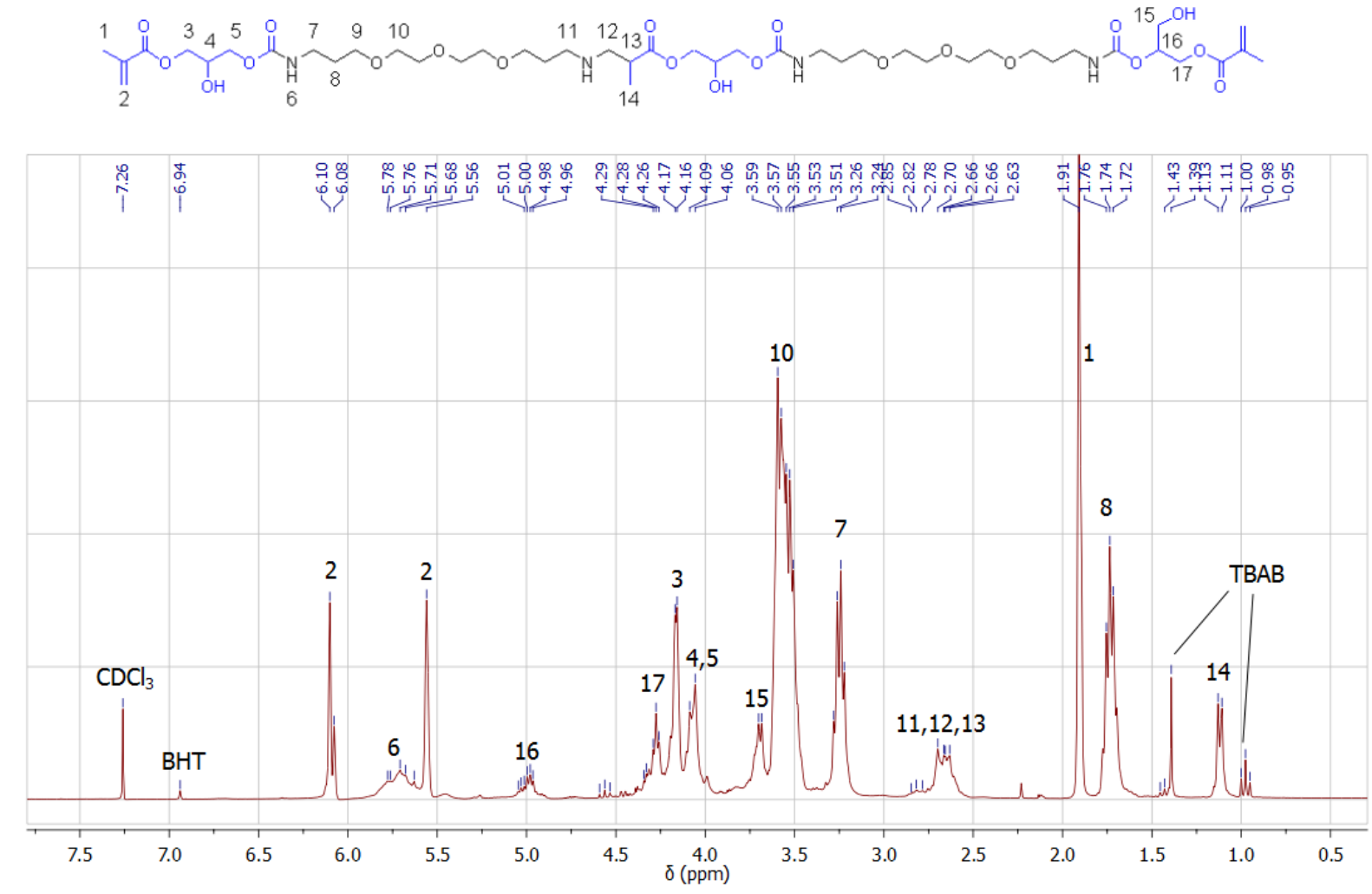

Figure S 5: ${ }^{1} \mathrm{H}-\mathrm{NMR}$ spectrum $\left(\mathrm{CDCl}_{3}\right)$ of TODA-G.

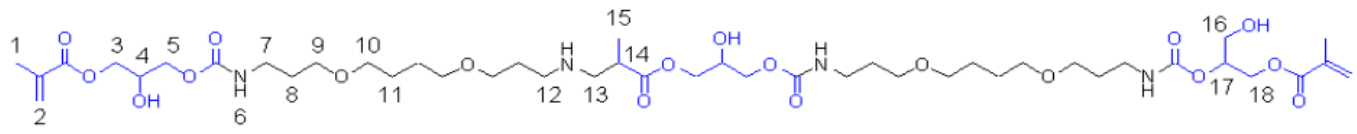

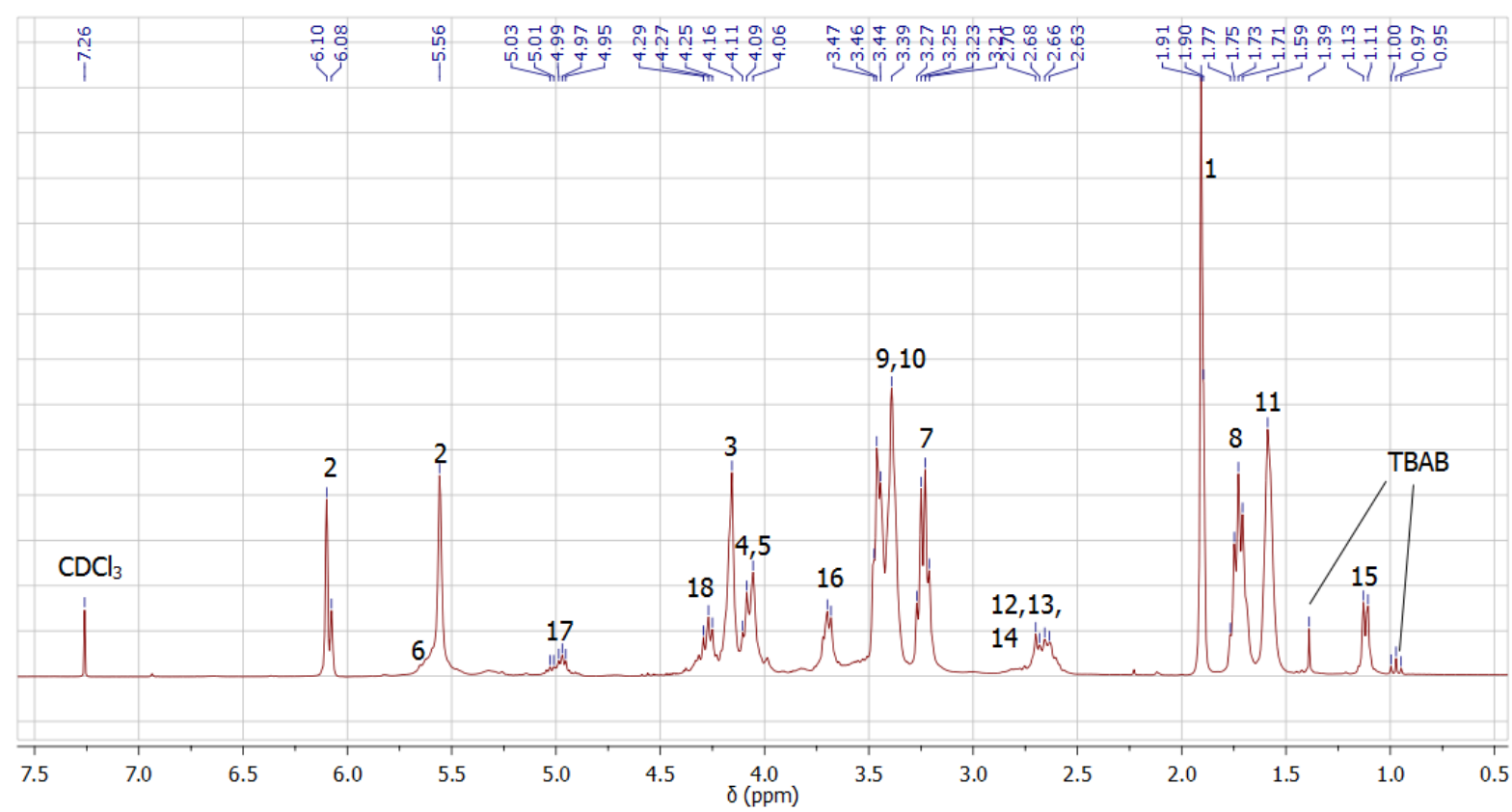

Figure S 6: ${ }^{1} \mathrm{H}-\mathrm{NMR}$ spectrum $\left(\mathrm{CDCl}_{3}\right)$ of DODA12-G. 


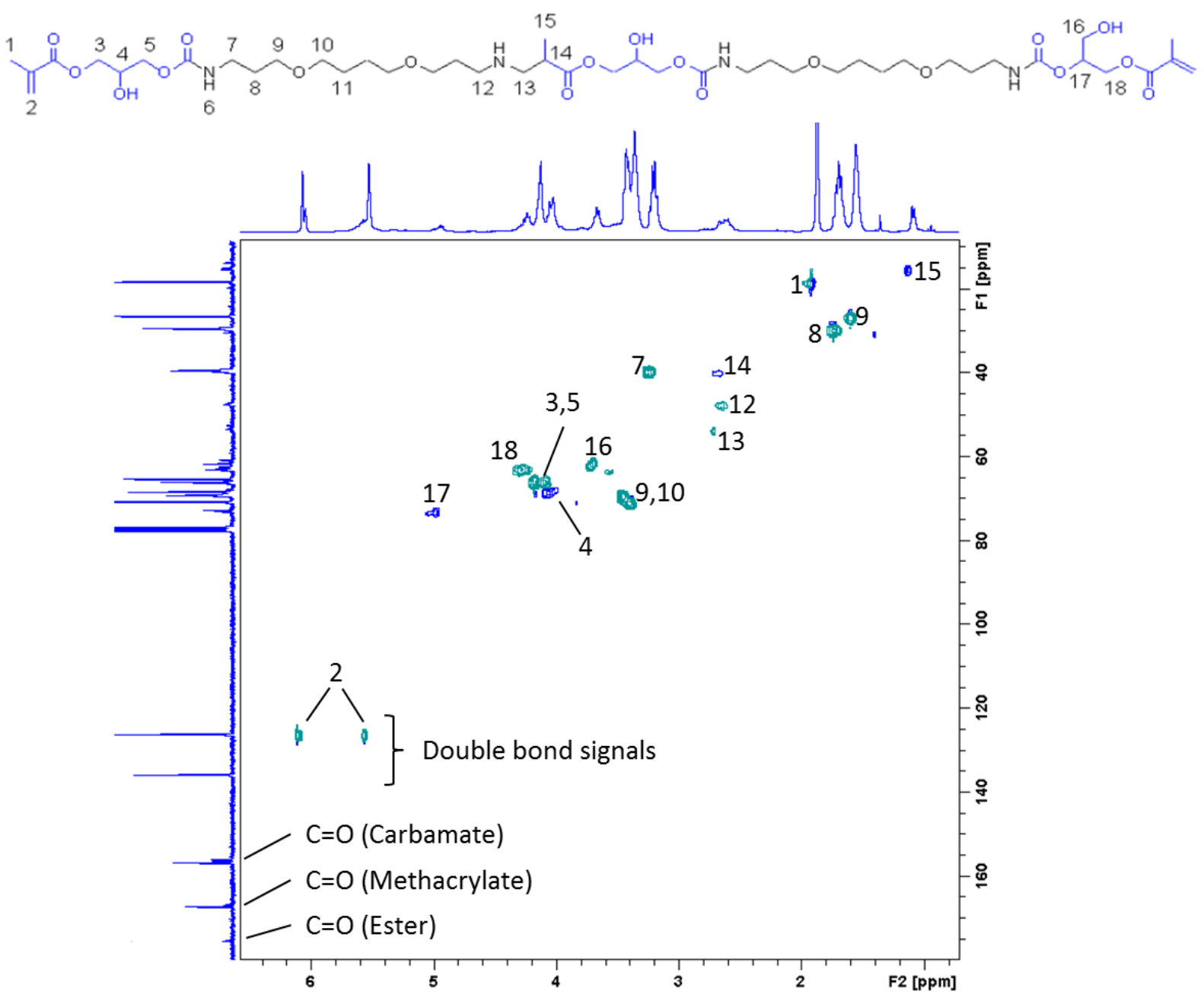

Figure S 7: ${ }^{1} \mathrm{H} /{ }^{13} \mathrm{C}-\mathrm{HSQC}-\mathrm{NMR}$ spectrum $\left(\mathrm{CDCl}_{3}\right)$ of DODA12-G. 


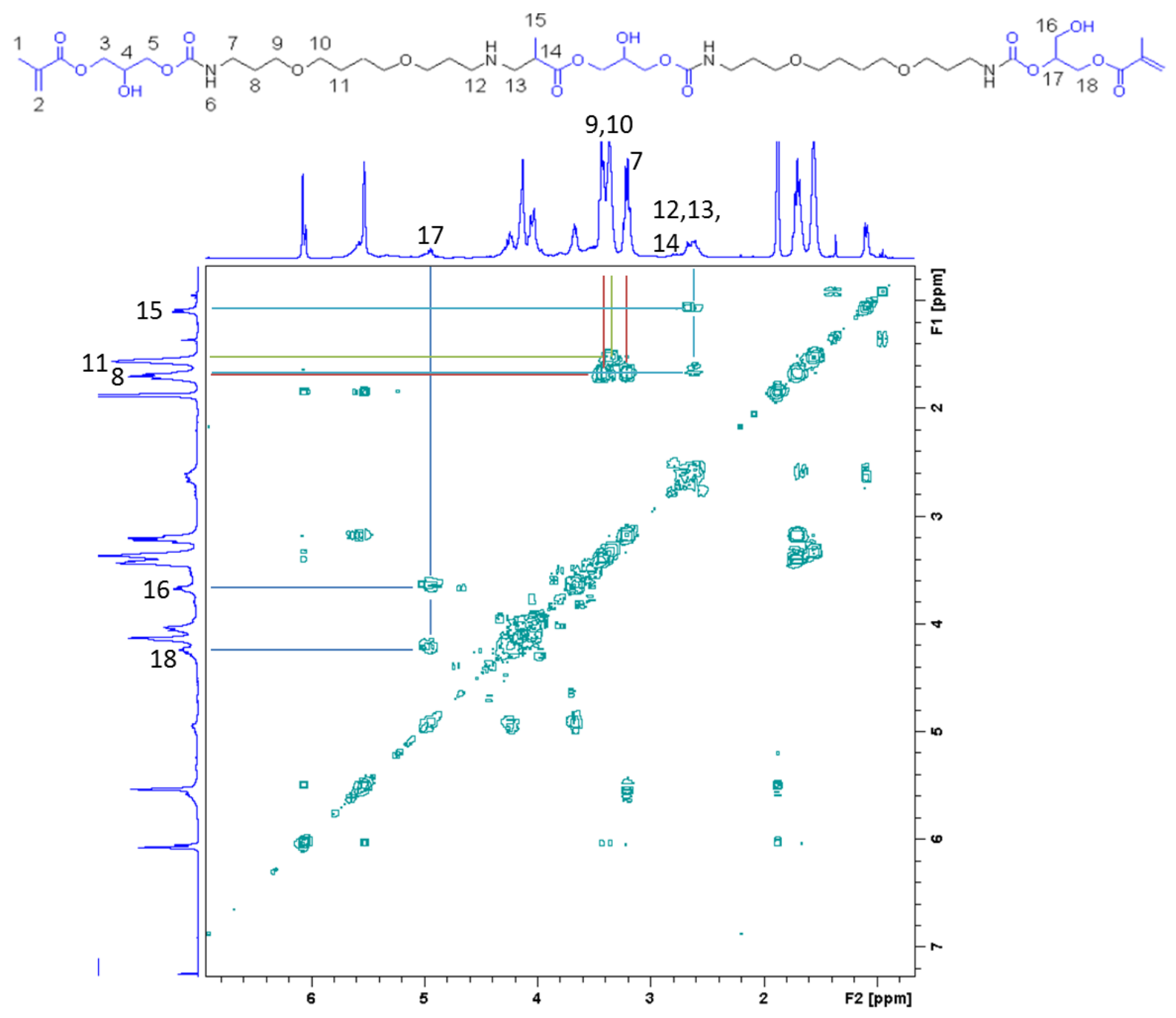

Figure S 8: ${ }^{1} \mathrm{H} /{ }^{1} \mathrm{H}-\mathrm{COSY}-\mathrm{NMR}\left(\mathrm{CDCl}_{3}\right)$ spectrum of DODA12-G. 


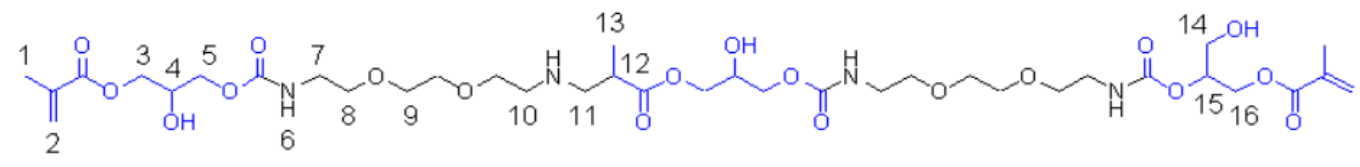

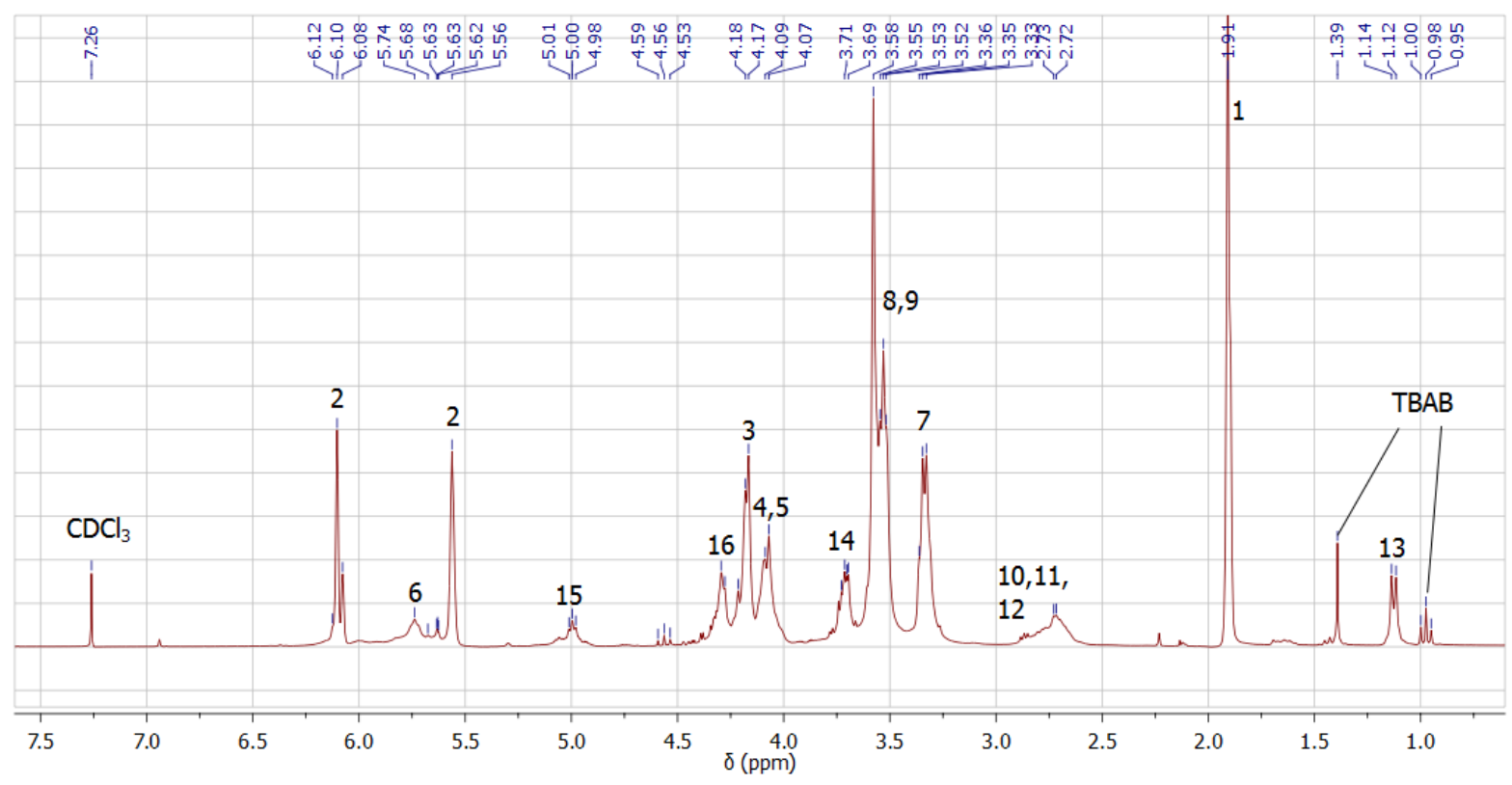

Figure S 9: ${ }^{1} \mathrm{H}-\mathrm{NMR}$ spectrum $\left(\mathrm{CDCl}_{3}\right)$ of DODA8-G.
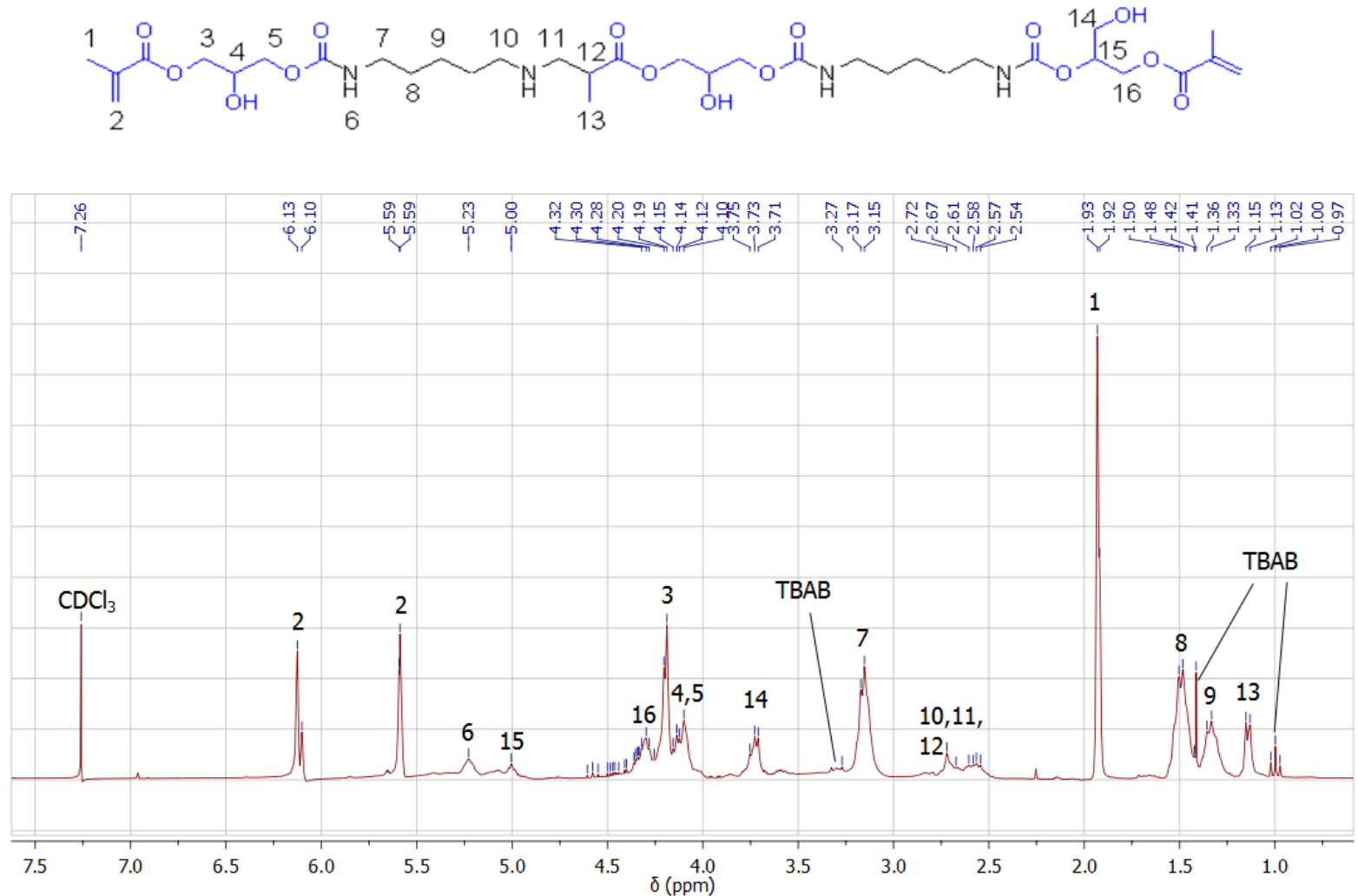

Figure S 10: ${ }^{1} \mathrm{H}-\mathrm{NMR}$ spectrum $\left(\mathrm{CDCl}_{3}\right)$ of DAP-G. 

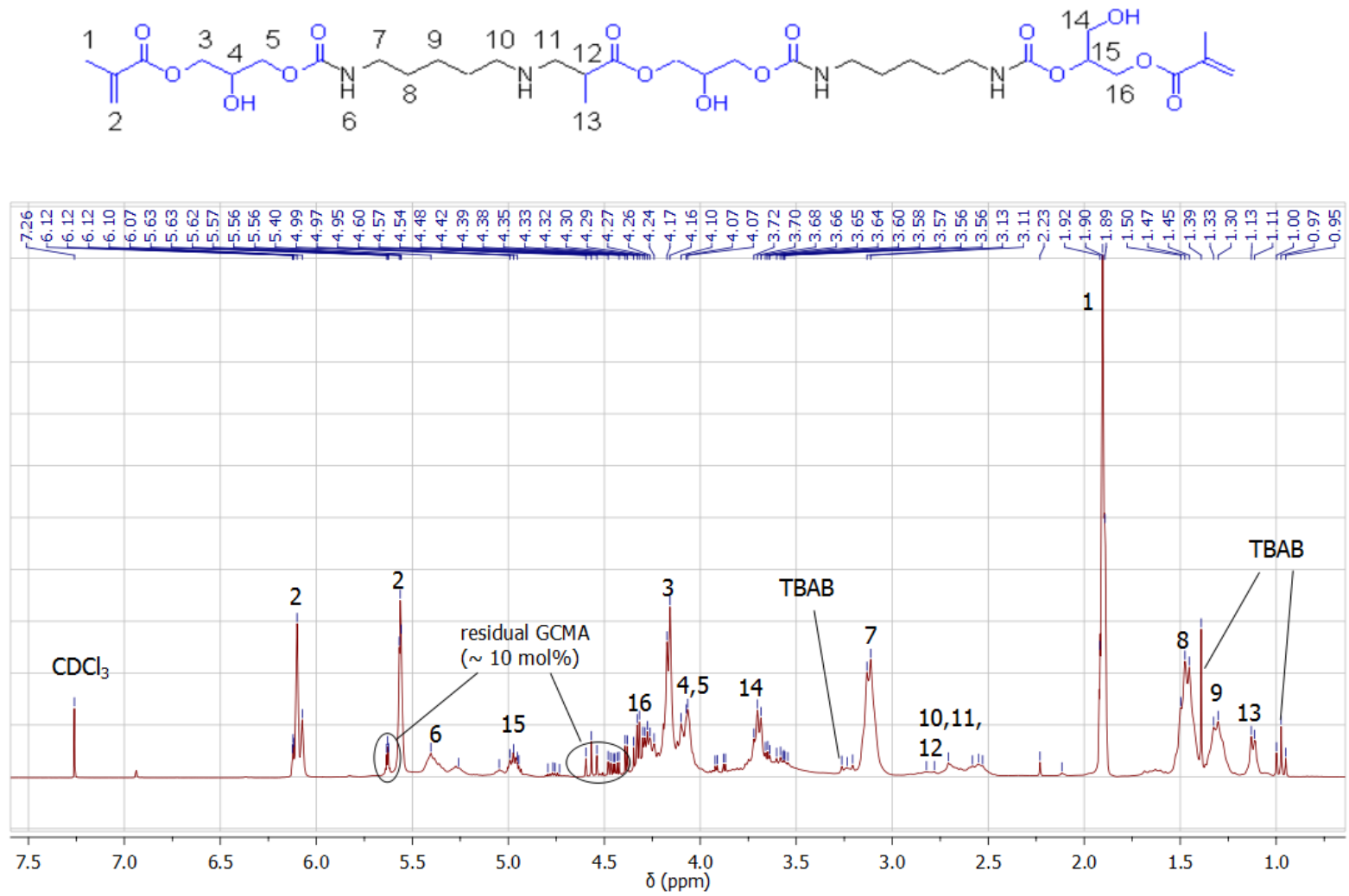

Figure S 11: ${ }^{1} \mathrm{H}-\mathrm{NMR}$ spectrum $\left(\mathrm{CDCl}_{3}\right)$ of DAP-G-60.

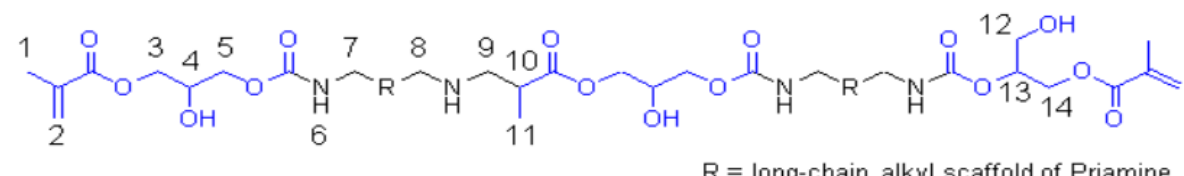

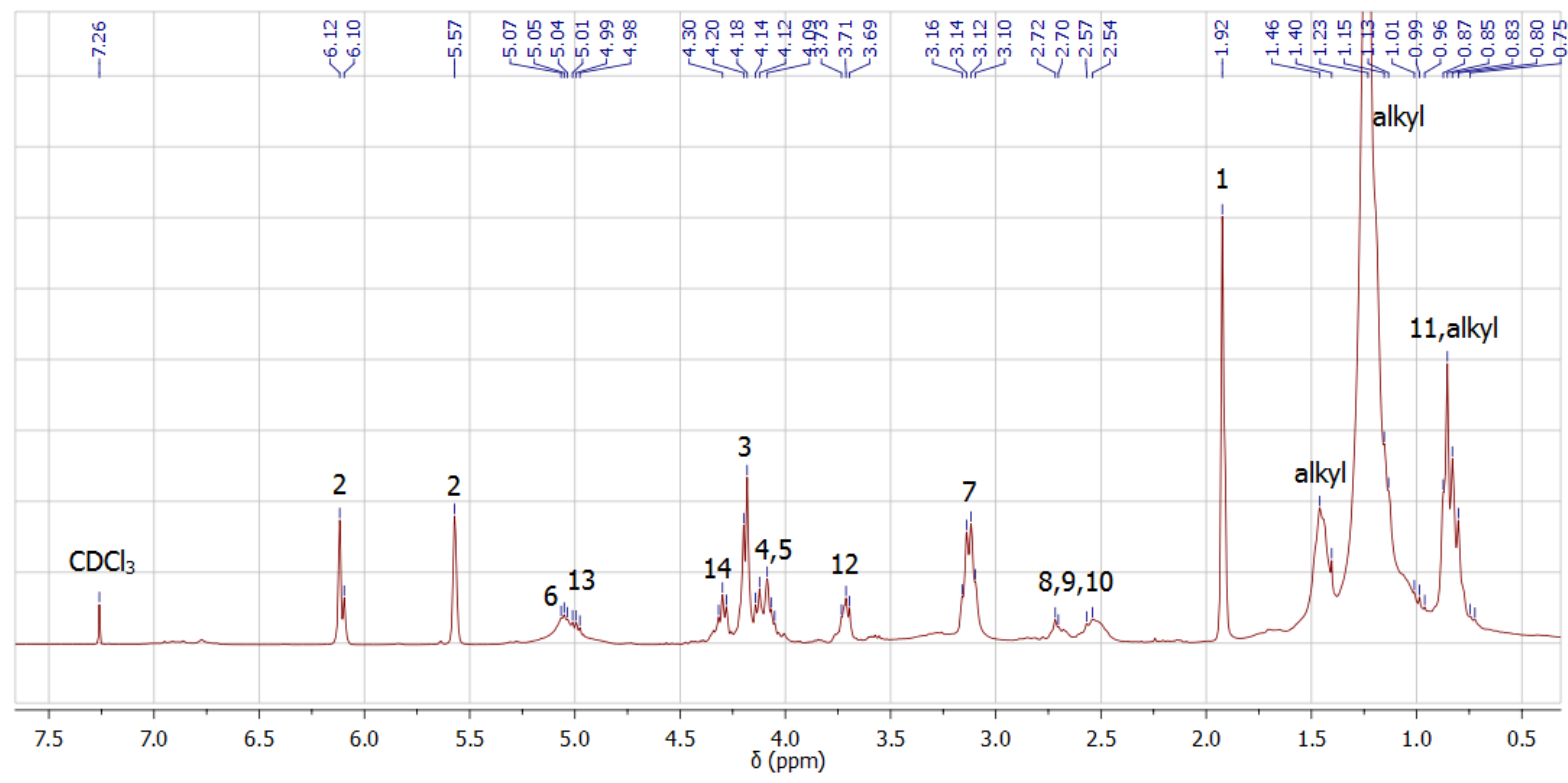

Figure S 12: ${ }^{1} \mathrm{H}-\mathrm{NMR}$ spectrum $\left(\mathrm{CDCl}_{3}\right)$ of PA-G. 


$$
\prod_{2}^{1} \overbrace{\mathrm{OH}}^{\mathrm{I}_{2}}
$$

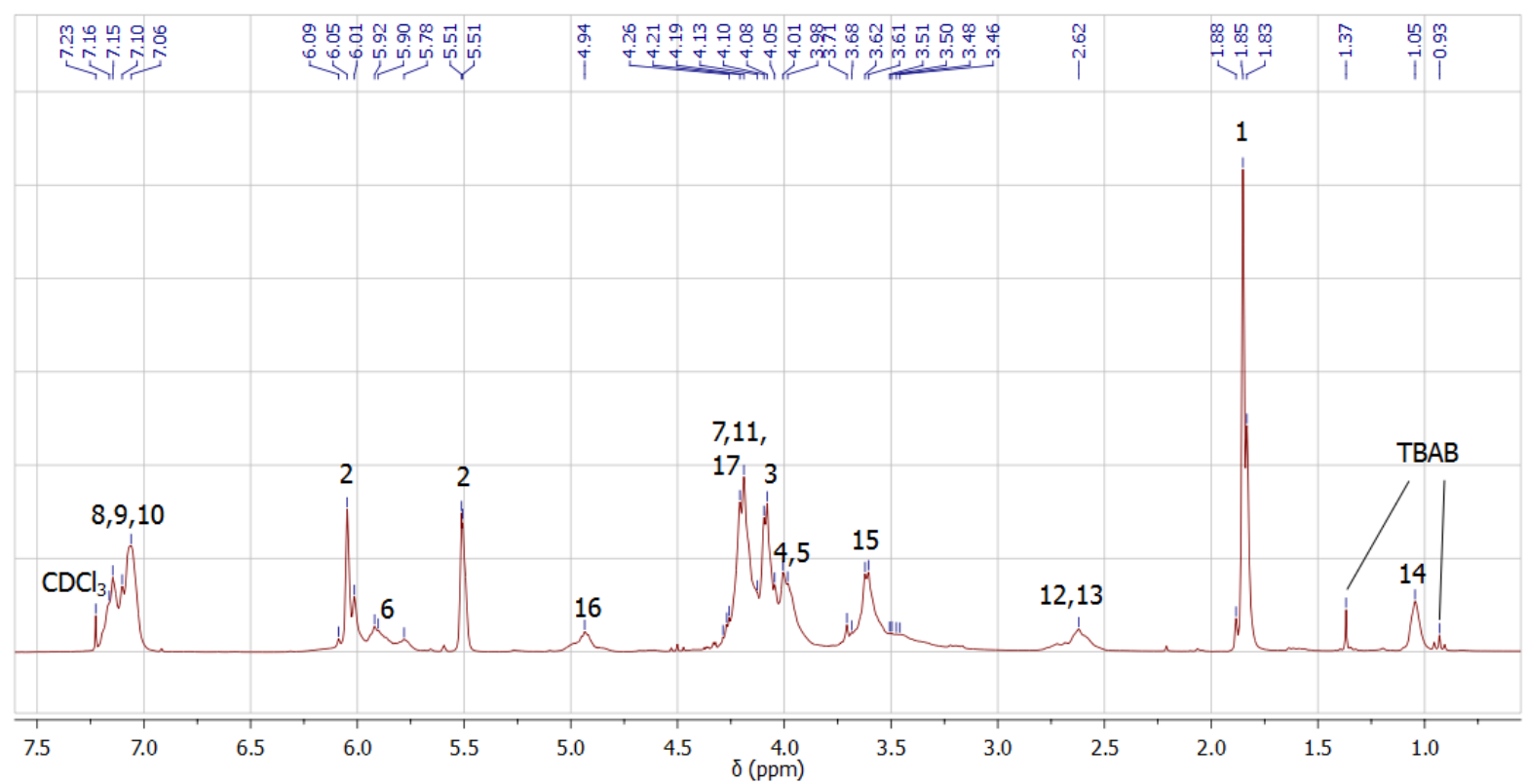

Figure $\mathbf{S}$ 13: ${ }^{1} \mathrm{H}-\mathrm{NMR}$ spectrum $\left(\mathrm{CDCl}_{3}\right)$ of XDA-G.

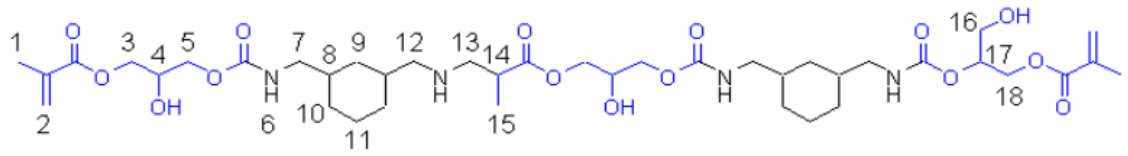

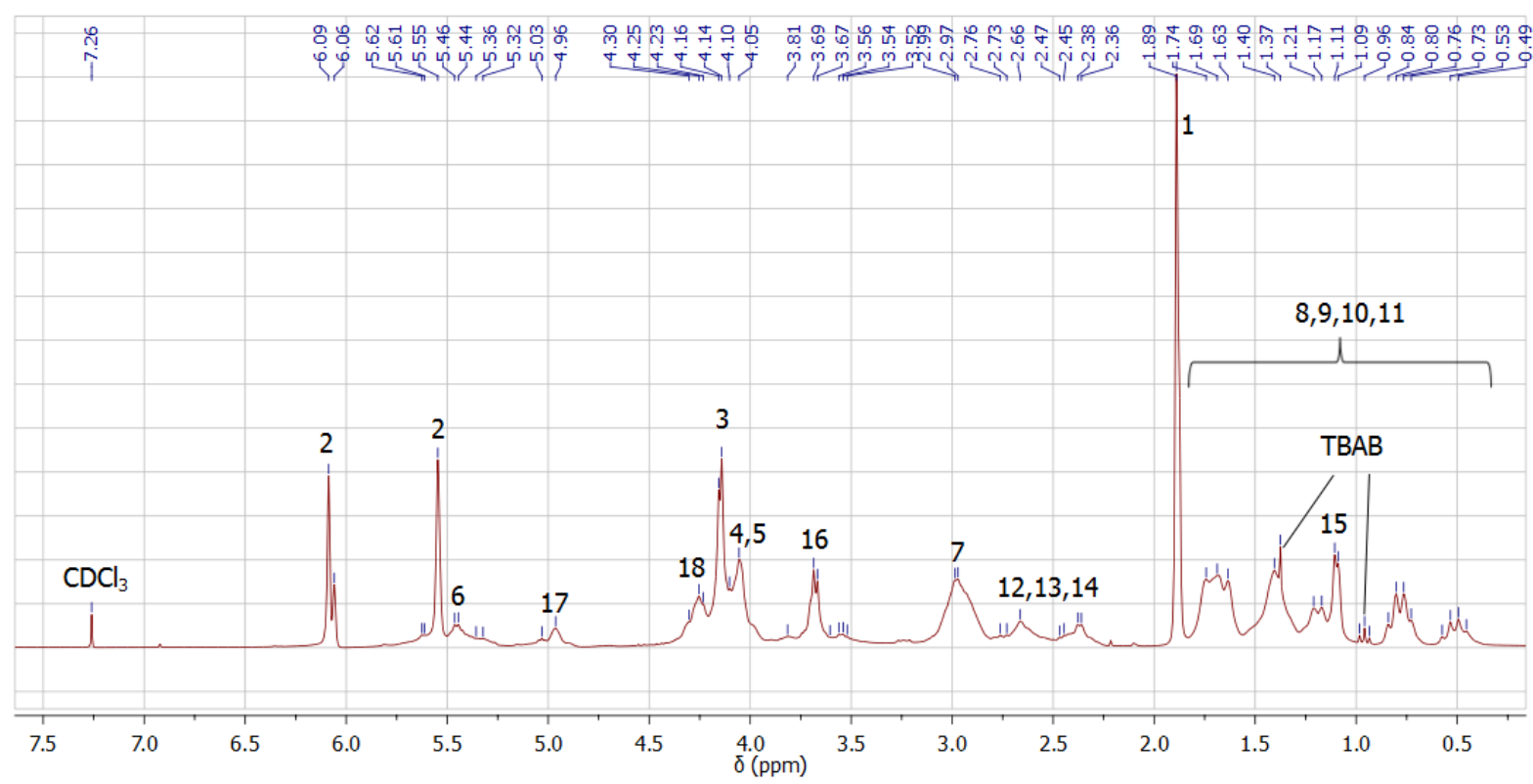

Figure S 14: ${ }^{1} \mathrm{H}-\mathrm{NMR}$ spectrum $\left(\mathrm{CDCl}_{3}\right)$ of CDMA-G. 


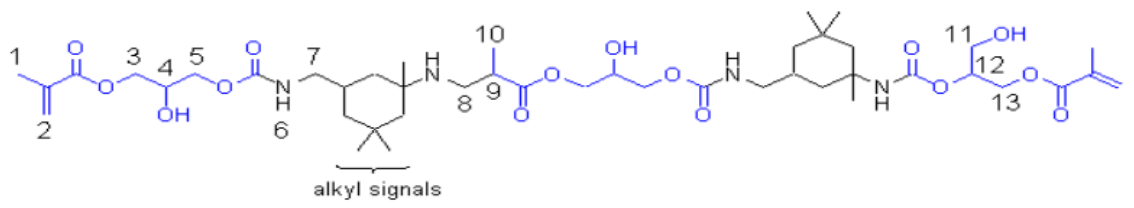

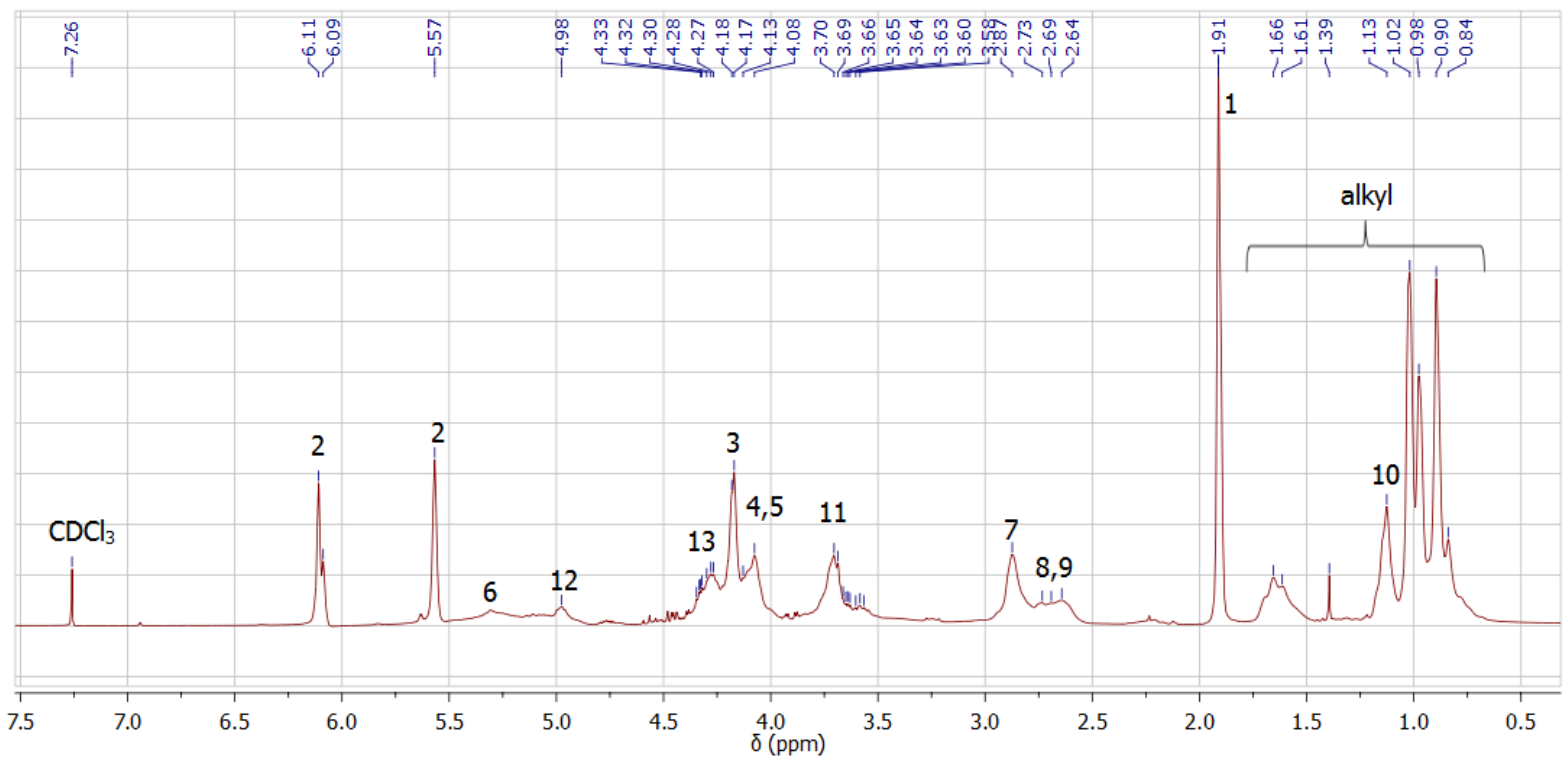

Figure S 15: ${ }^{1} \mathrm{H}-\mathrm{NMR}$ spectrum $\left(\mathrm{CDCl}_{3}\right)$ of IPDA-G.

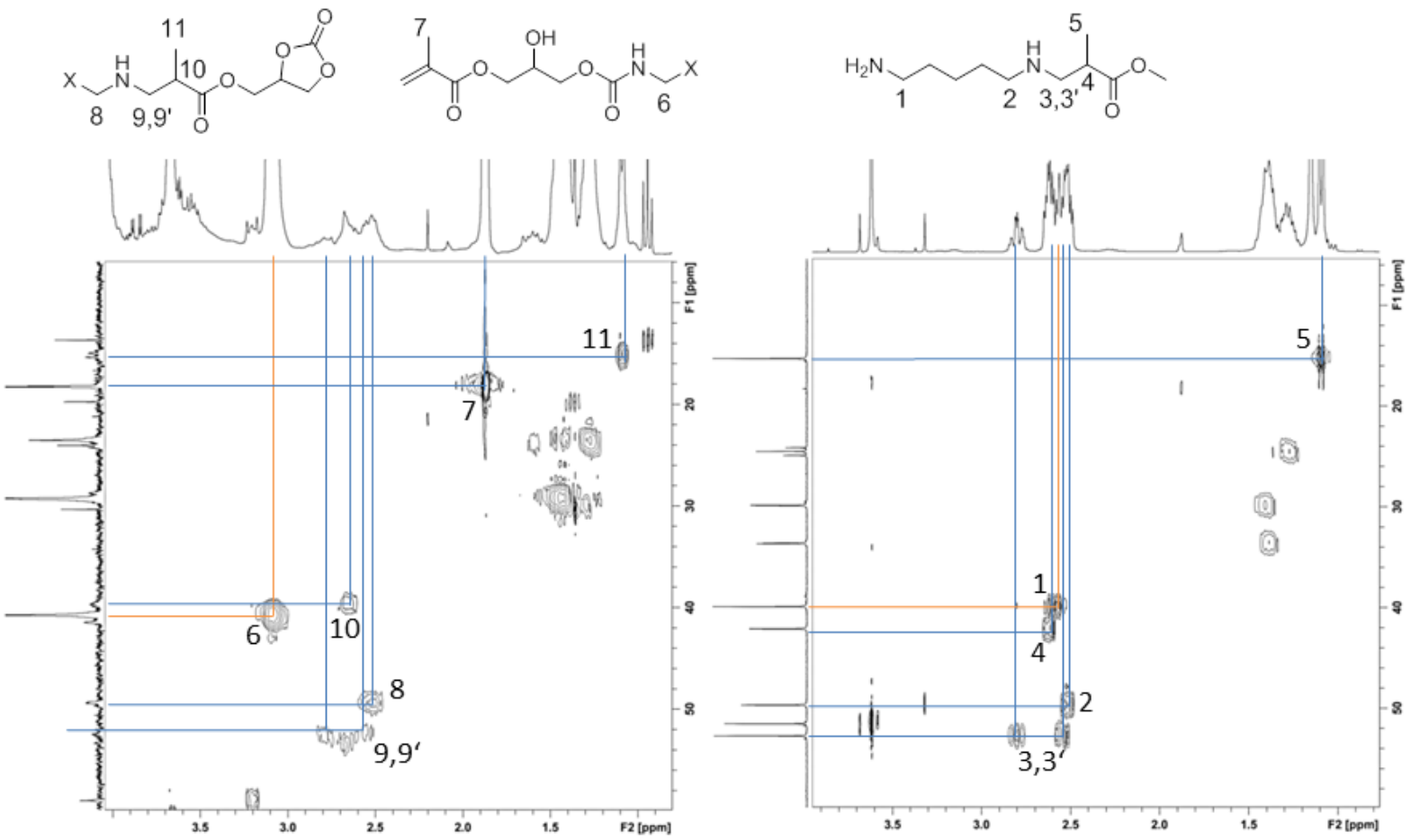

Figure S 16: ${ }^{1} \mathrm{H} /{ }^{13} \mathrm{C}-\mathrm{HSQC}-\mathrm{NMR}$ spectra $\left(\mathrm{CDCl}_{3}\right)$ of DAP-G-60 (left) and DAP_MMA (right). 


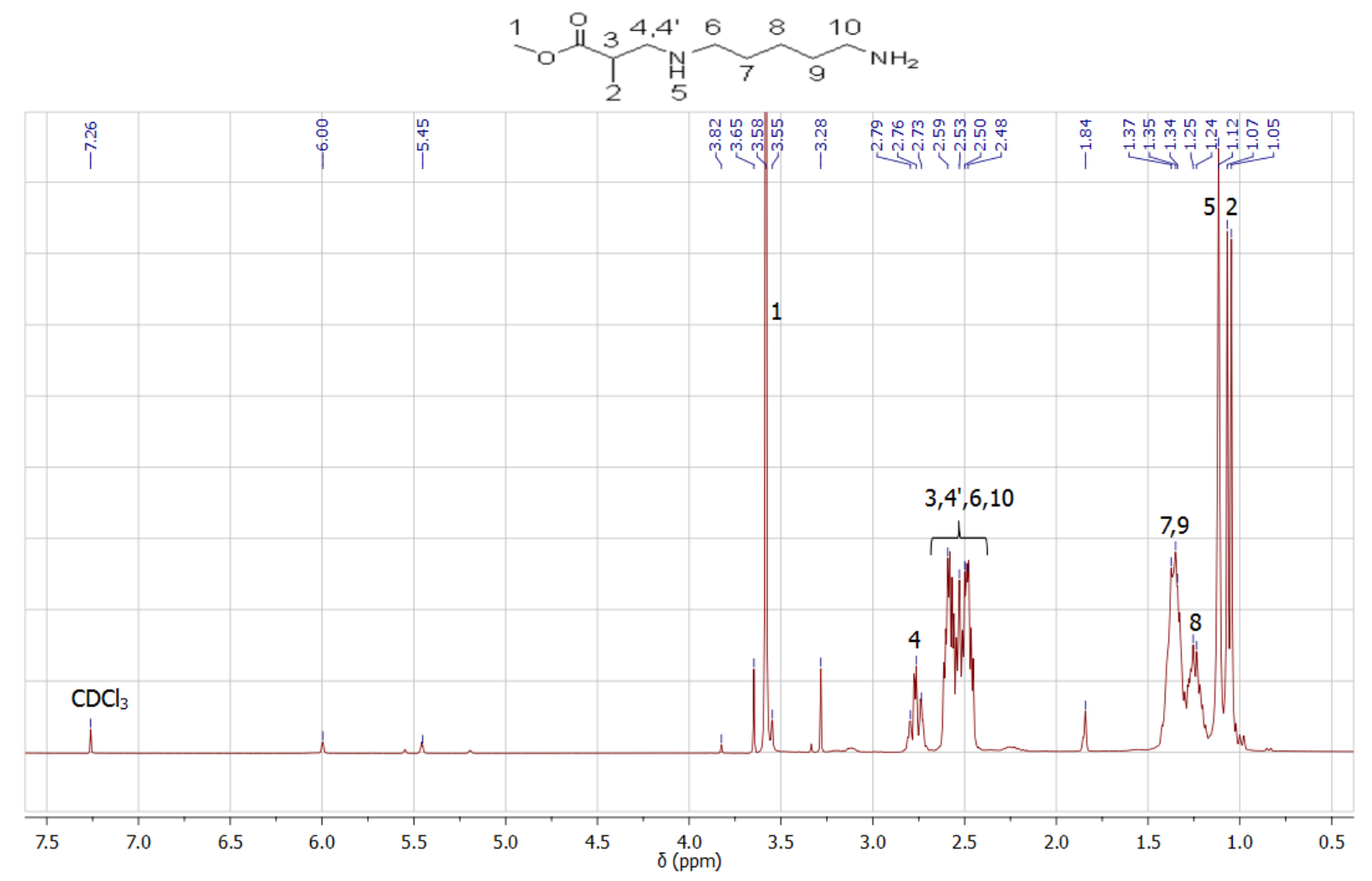

Figure S 17: ${ }^{1} \mathrm{H}-\mathrm{NMR}$ spectrum $\left(\mathrm{CDCl}_{3}\right)$ of DAP_MMA.
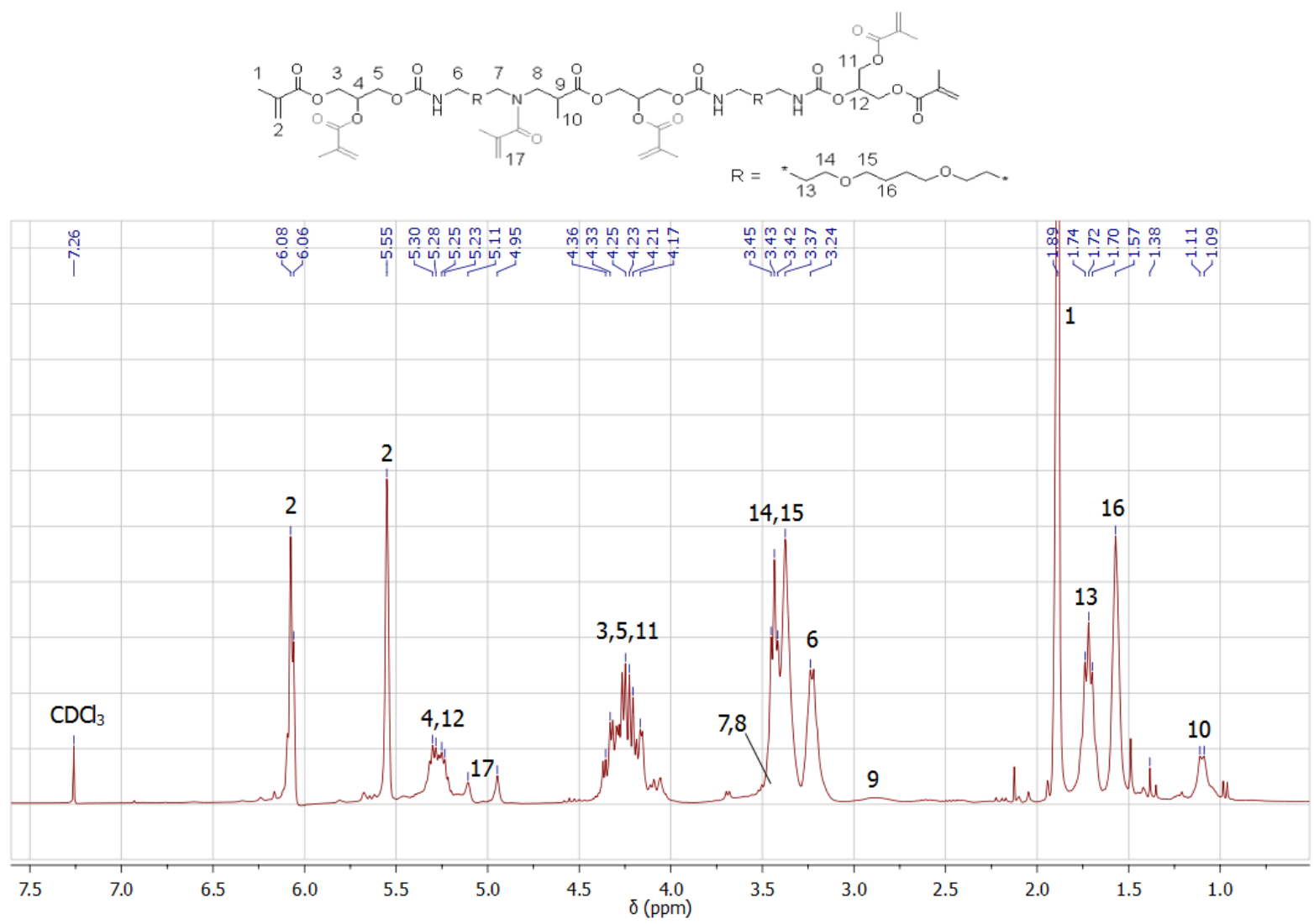

Figure S 18: ${ }^{1} \mathrm{H}-\mathrm{NMR}$ spectrum $\left(\mathrm{CDCl}_{3}\right)$ of DODA12-G5. 


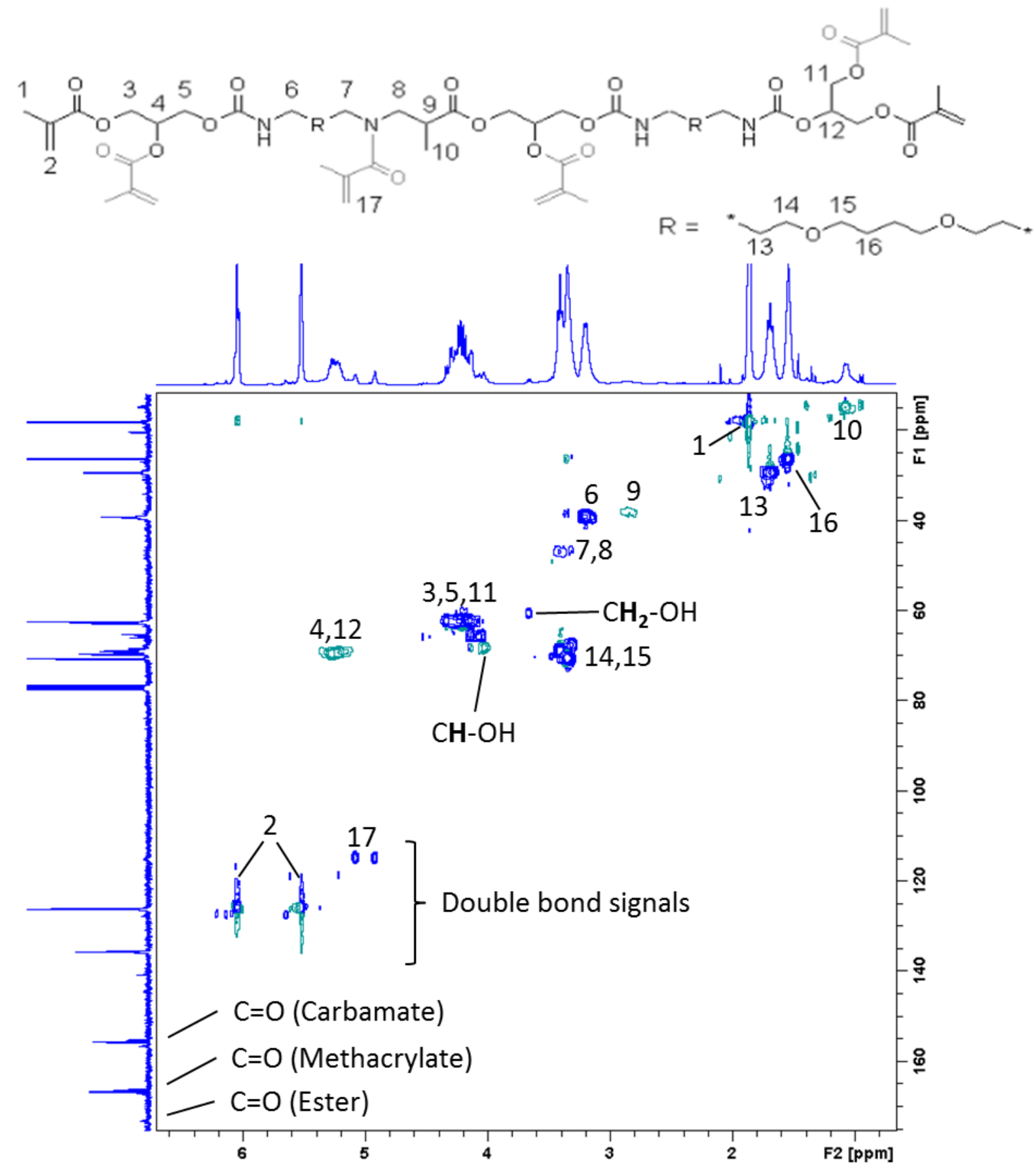

Figure S 19: ${ }^{1} \mathrm{H} /{ }^{13} \mathrm{C}-\mathrm{HSQC}-\mathrm{NMR}$ spectrum $\left(\mathrm{CDCl}_{3}\right)$ of DODA12-G5. 


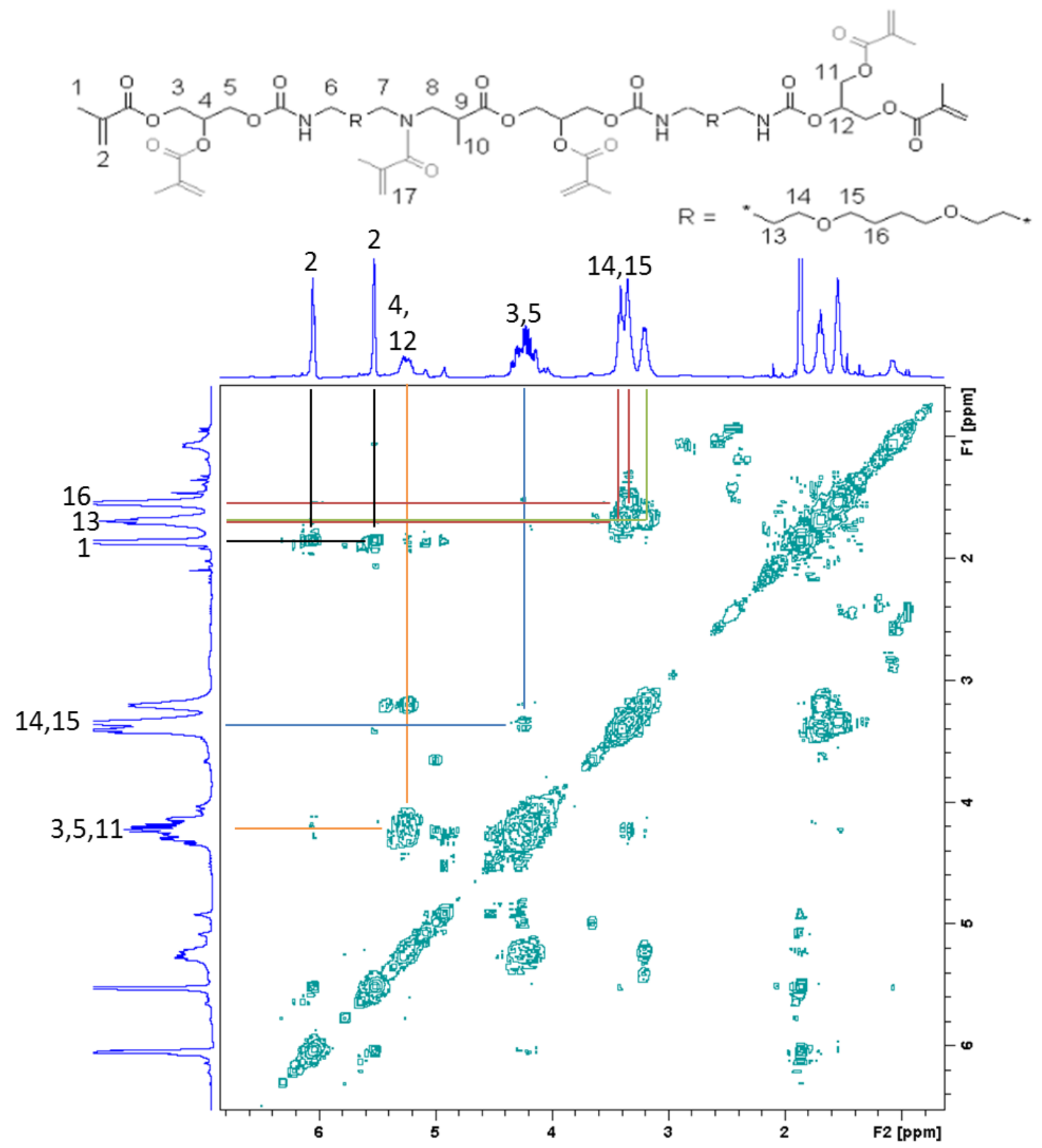

Figure S 20: ${ }^{1} \mathrm{H} /{ }^{1} \mathrm{H}-\mathrm{COSY}-\mathrm{NMR}\left(\mathrm{CDCl}_{3}\right)$ spectrum of DODA12-G5. 


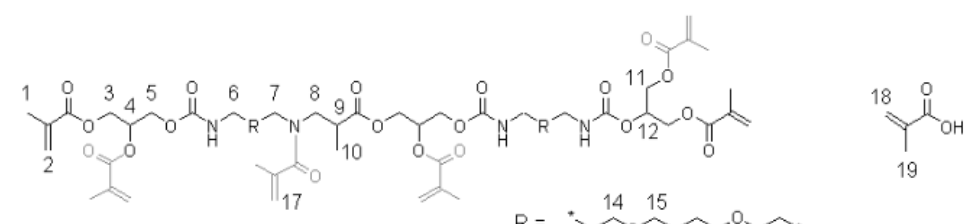

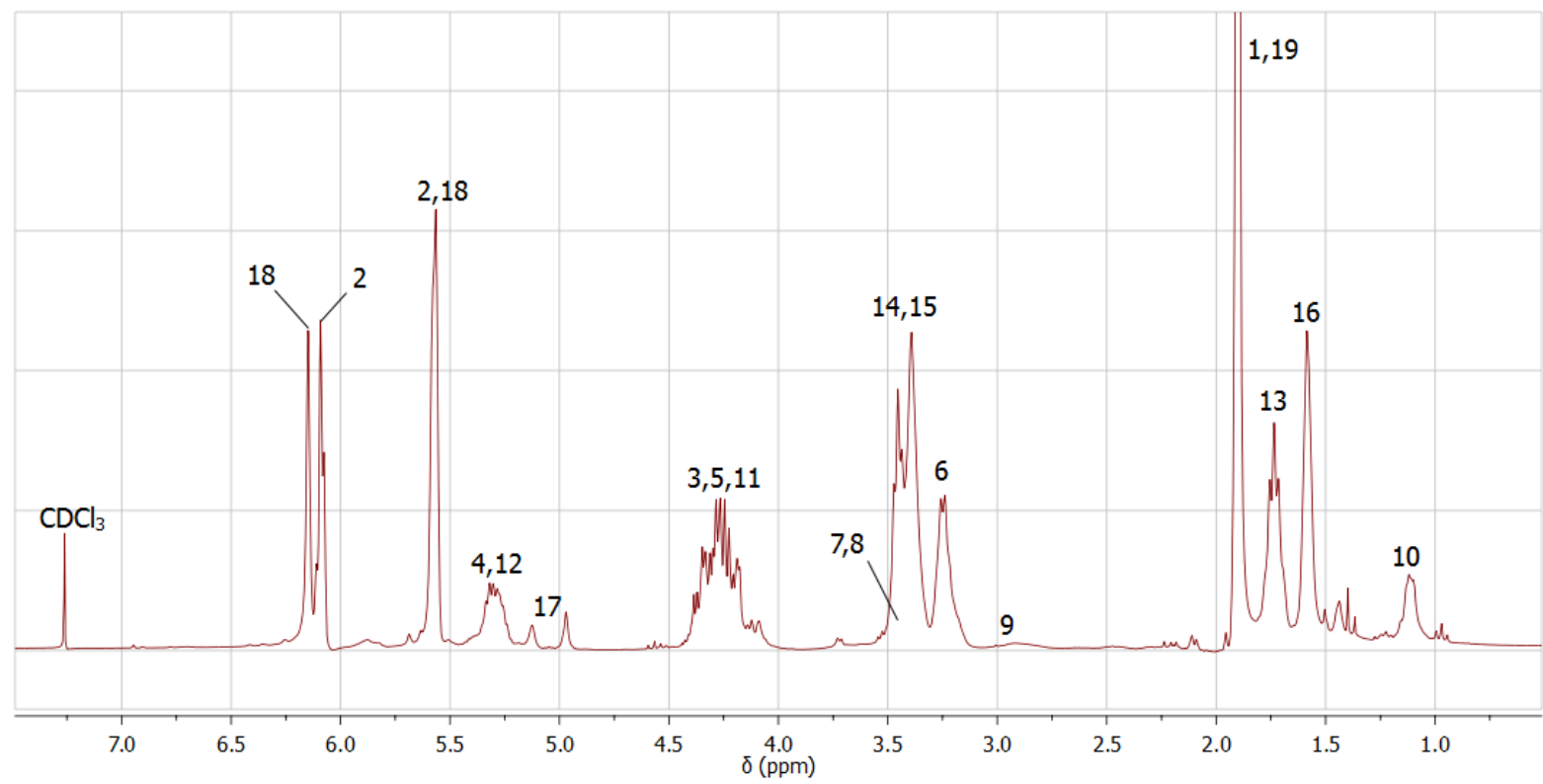

Figure S 21: ${ }^{1} \mathrm{H}-\mathrm{NMR}$ spectrum $\left(\mathrm{CDCl}_{3}\right)$ of DODA12-G5/MA.

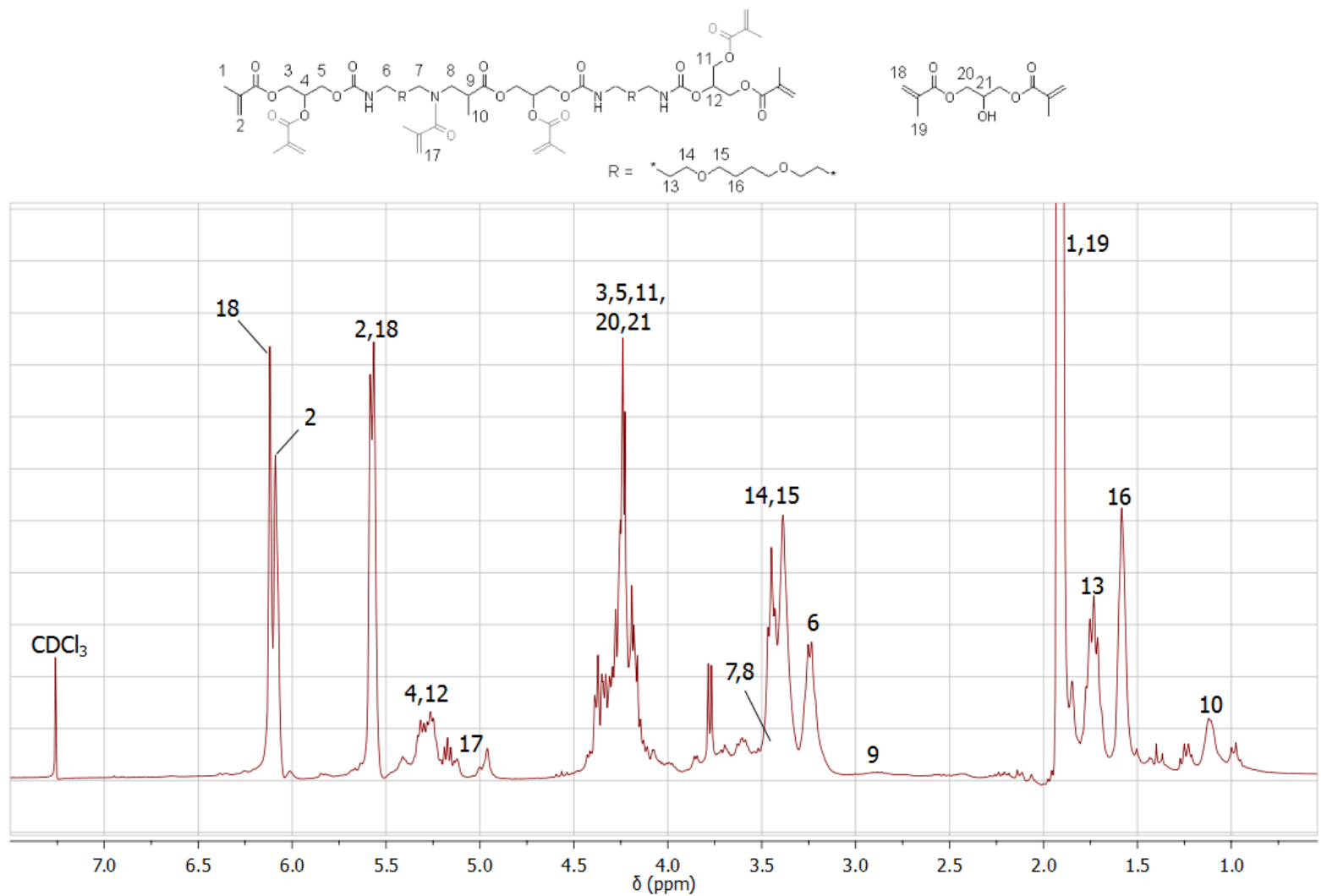

Figure S 22: ${ }^{1} \mathrm{H}-\mathrm{NMR}$ spectrum $\left(\mathrm{CDCl}_{3}\right)$ of DODA12-G5/GDMA. 


\section{II) DSC graphs}

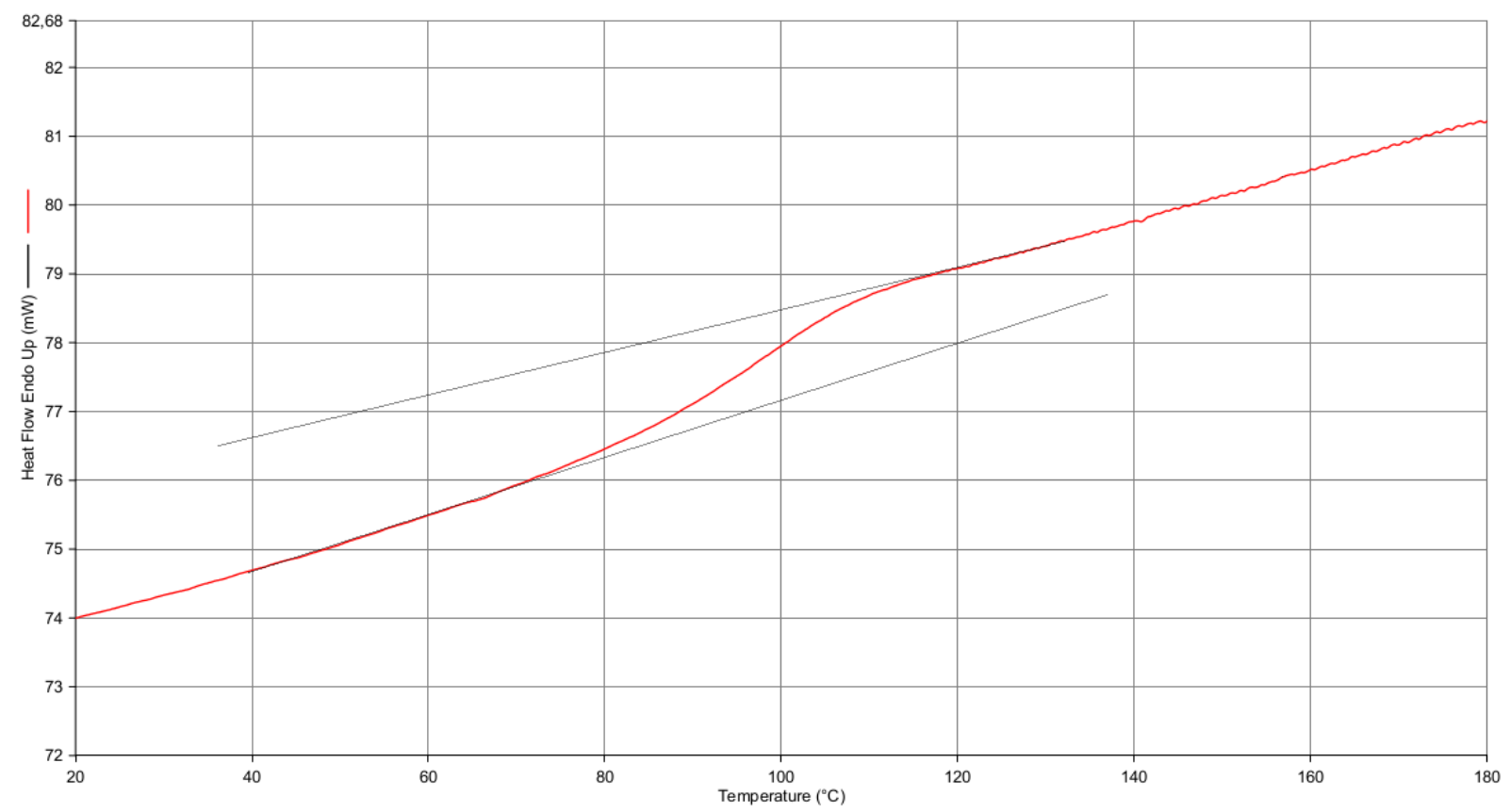

Figure S 23: DSC graph $\left(2^{\text {nd }}\right.$ heating, $\left.0-200^{\circ} \mathrm{C}, 10 \mathrm{~K} \mathrm{~min}^{-1}\right)$ of $A C M O \_L a r o m e r$.

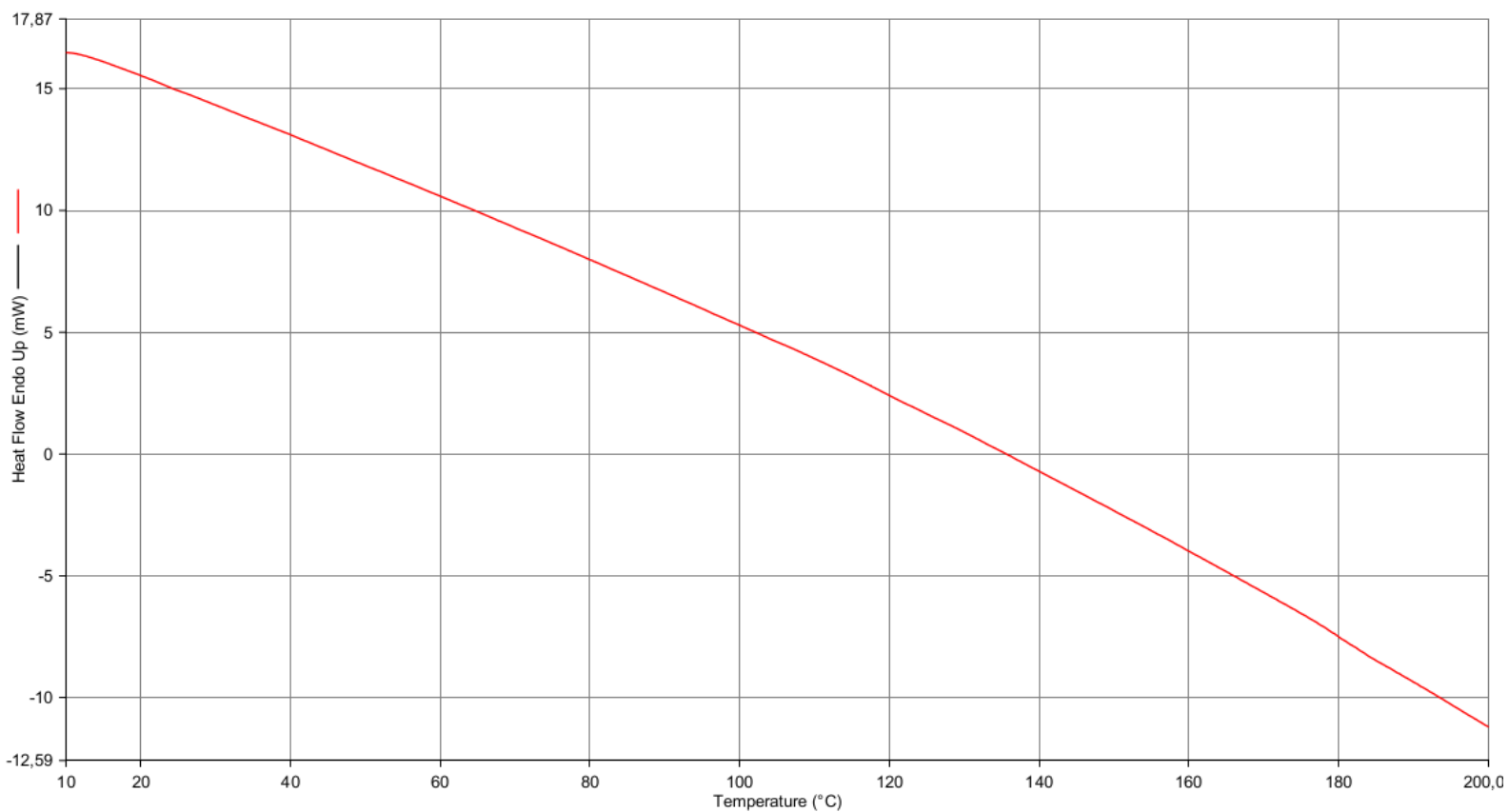

Figure S 24: DSC graph $\left(2^{\text {nd }}\right.$ heating, $\left.0-200^{\circ} \mathrm{C}, 10 \mathrm{~K} \mathrm{~min}^{-1}\right)$ of ACMO_PTHFA-G. 


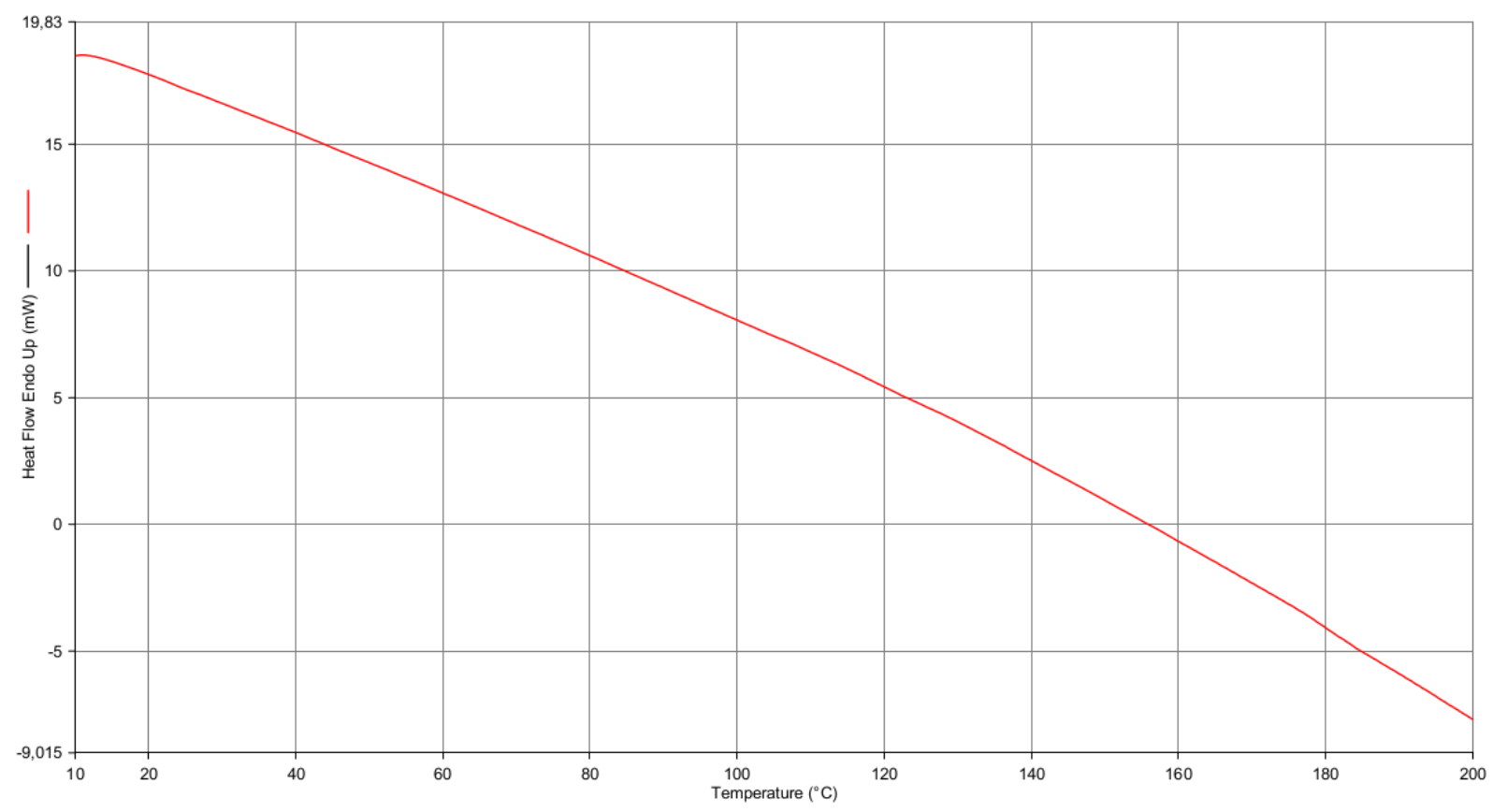

Figure S 25: DSC graph $\left(2^{\text {nd }}\right.$ heating, $\left.0-200^{\circ} \mathrm{C}, 10 \mathrm{~K} \mathrm{~min}^{-1}\right)$ of ACMO_JT3000-G.

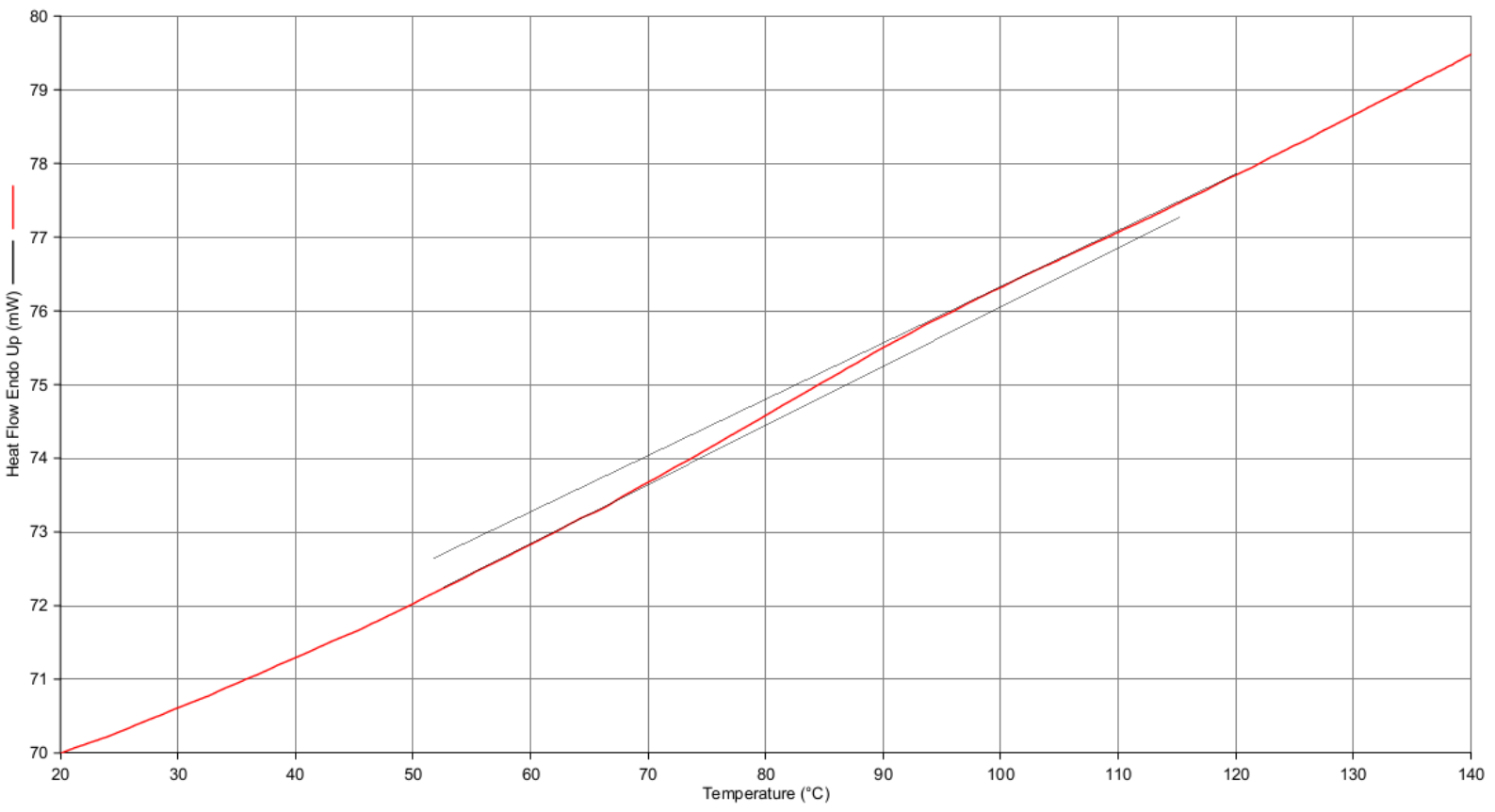

Figure S 26: DSC graph $\left(2^{\text {nd }}\right.$ heating, $\left.0-200^{\circ} \mathrm{C}, 10 \mathrm{~K} \mathrm{~min}^{-1}\right)$ of ACMO_JT403-G. 


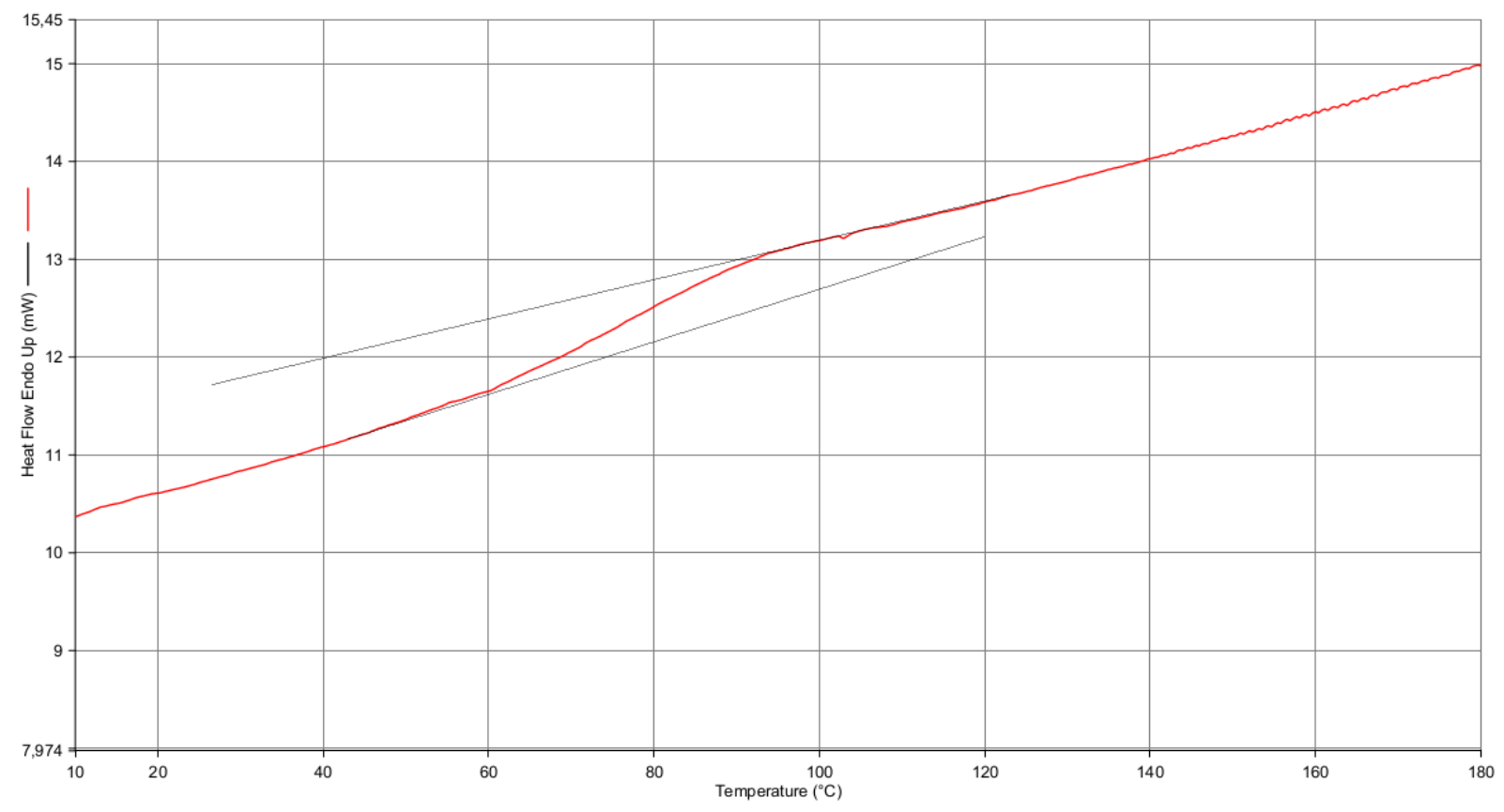

Figure S 27: DSC graph $\left(2^{\text {nd }}\right.$ heating, $\left.0-200^{\circ} \mathrm{C}, 10 \mathrm{~K} \mathrm{~min}^{-1}\right)$ of ACMO_TODA-G.

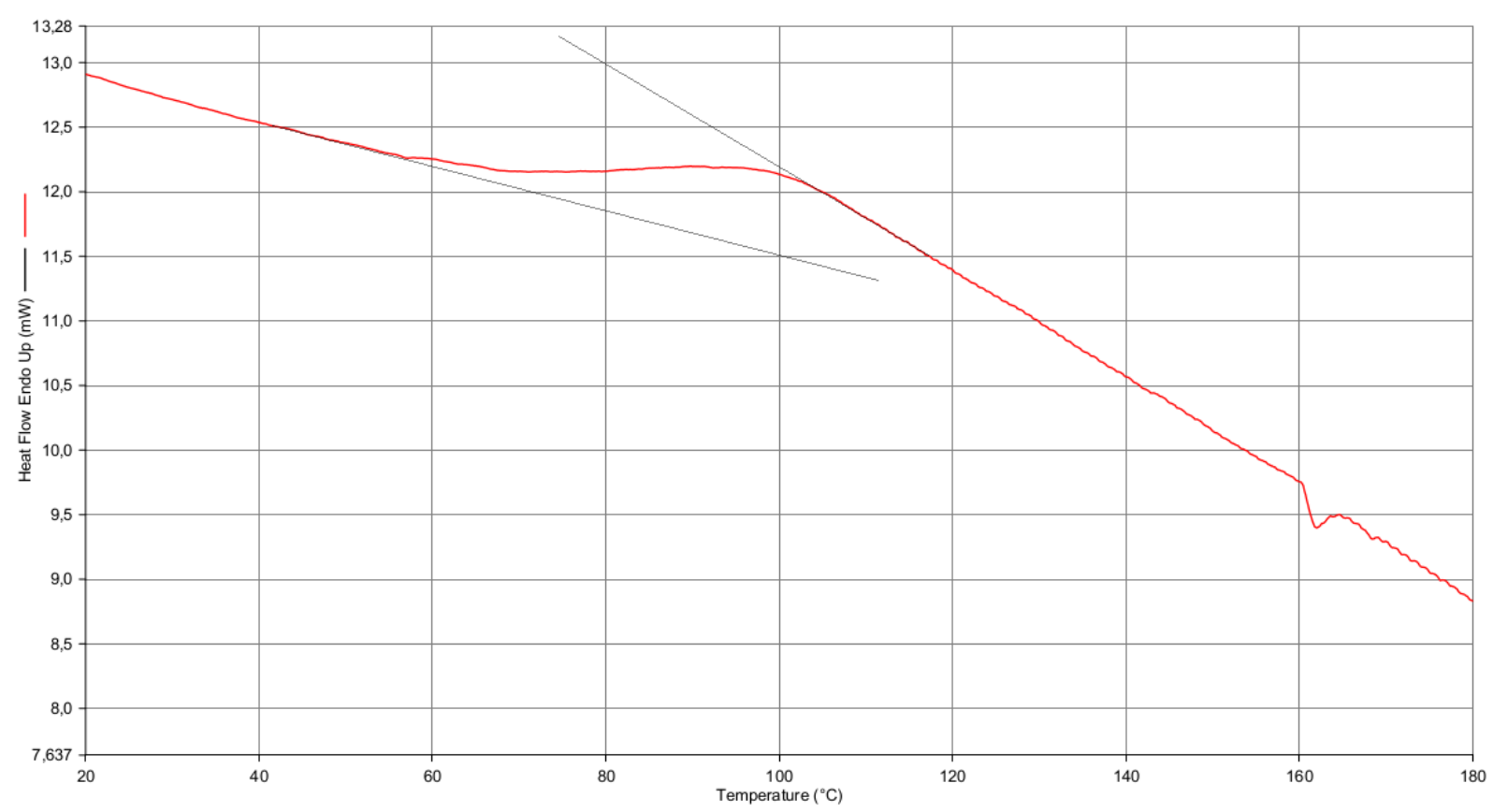

Figure S 28: DSC graph $\left(2^{\text {nd }}\right.$ heating, $\left.0-200^{\circ} \mathrm{C}, 10 \mathrm{~K} \mathrm{~min}^{-1}\right)$ of ACMO_DODA12 G. 


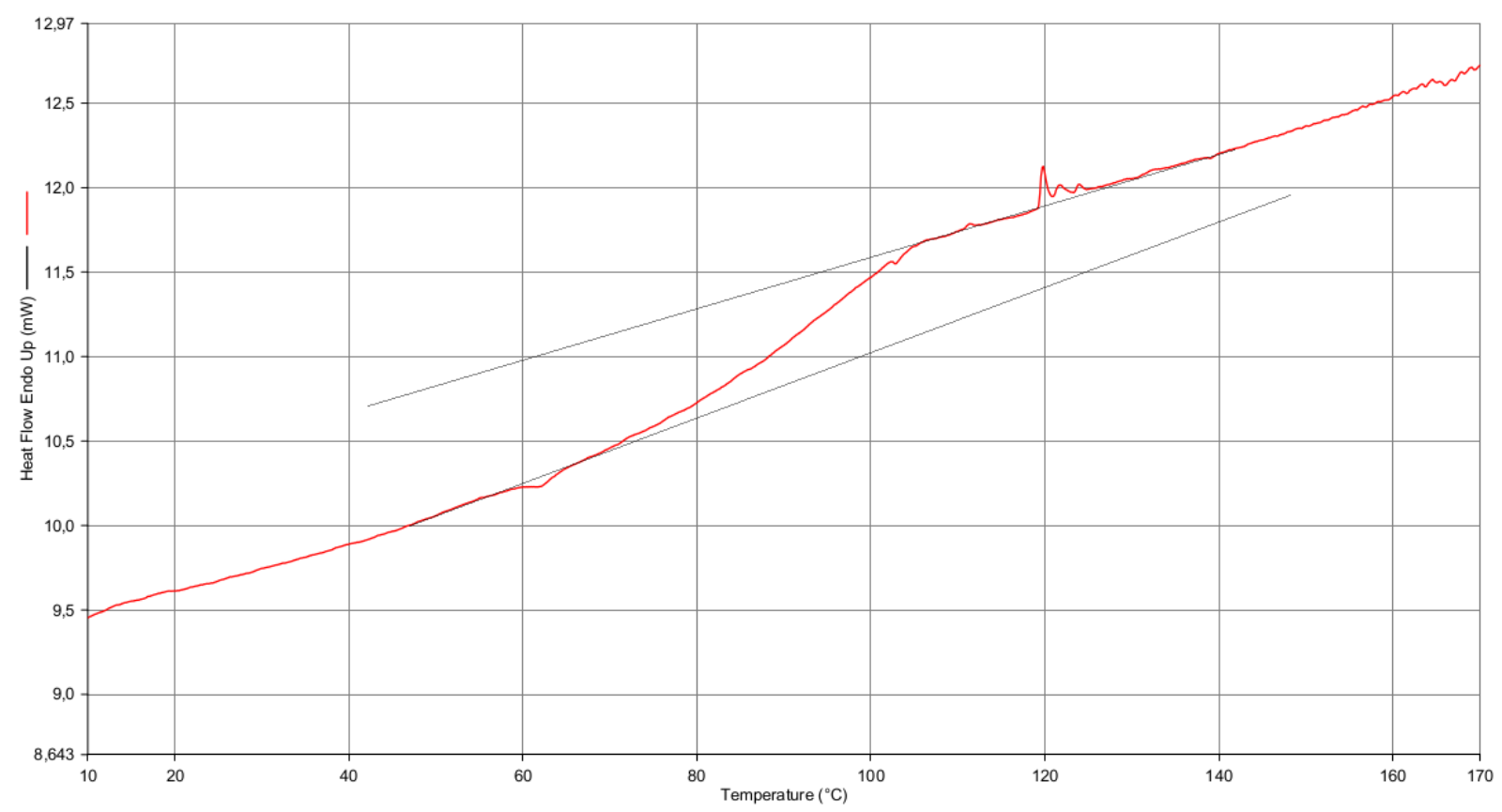

Figure S 29: DSC graph $\left(2^{\text {nd }}\right.$ heating, $\left.0-200^{\circ} \mathrm{C}, 10 \mathrm{~K} \mathrm{~min}{ }^{-1}\right)$ of ACMO_DODA8 G.

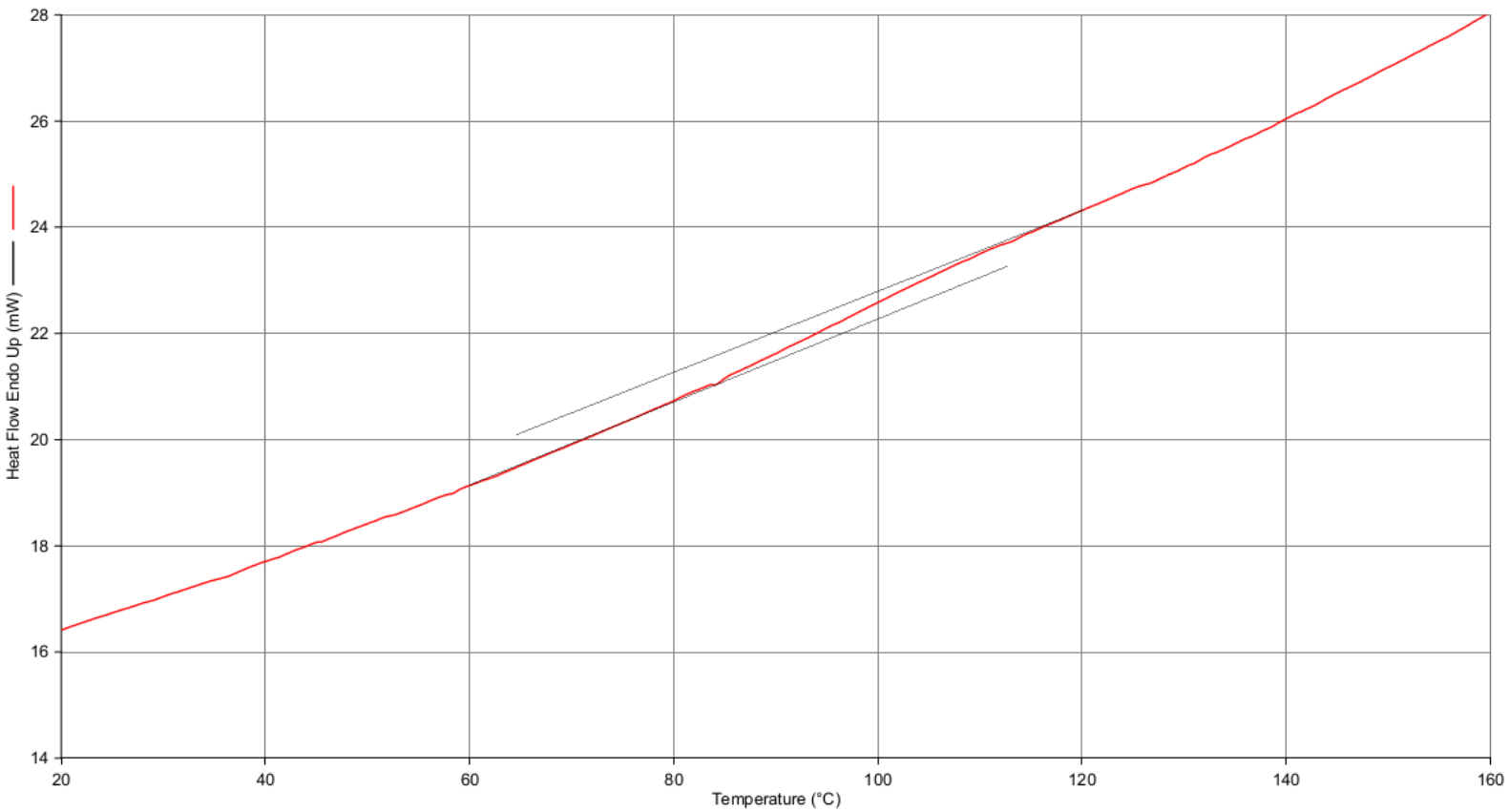

Figure S 30: DSC graph $\left(2^{\text {nd }}\right.$ heating, $\left.0-200^{\circ} \mathrm{C}, 10 \mathrm{~K} \mathrm{~min}^{-1}\right)$ of ACMO_PA-G. 


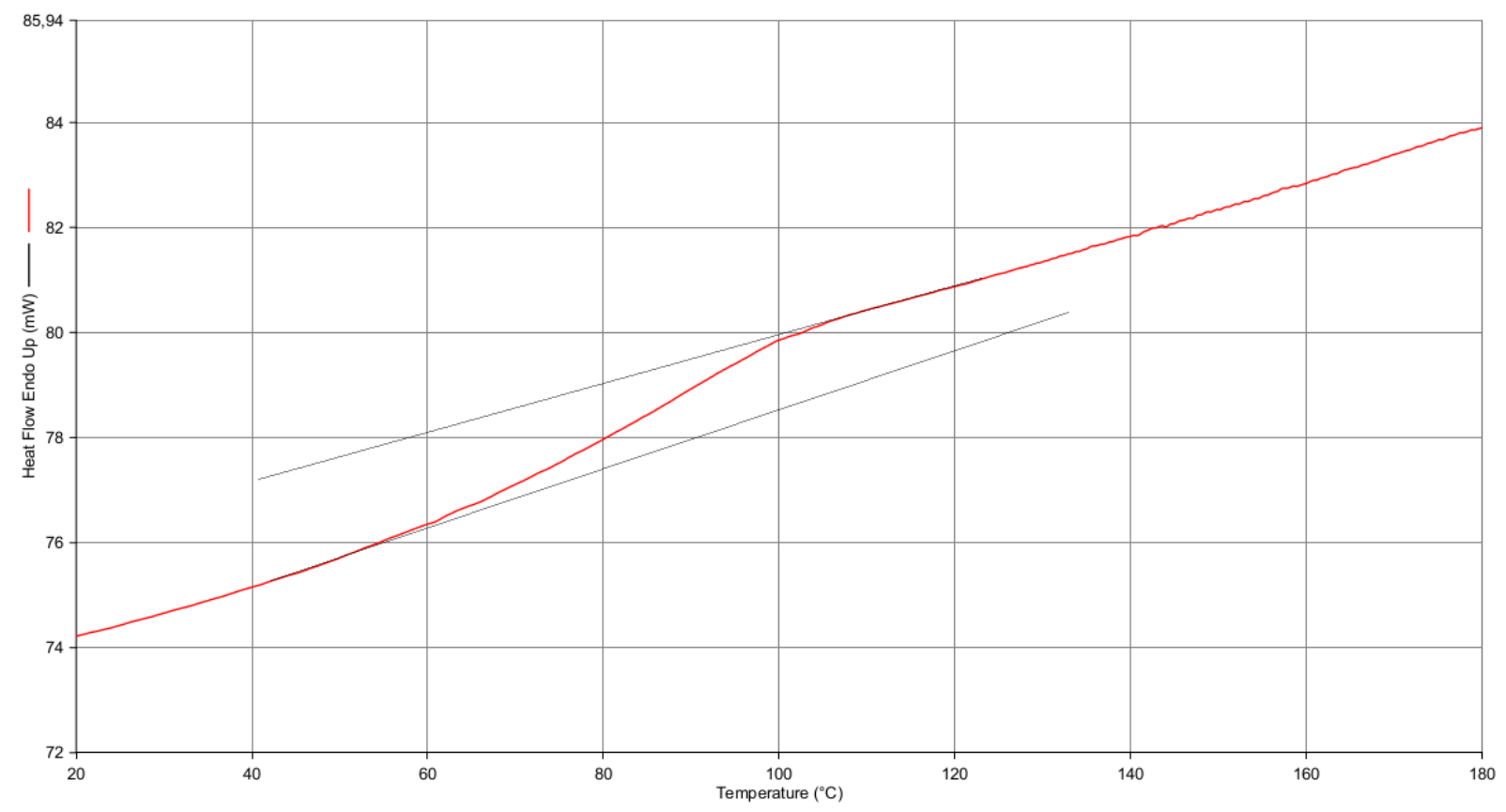

Figure S 31: DSC graph $\left(2^{\text {nd }}\right.$ heating, $\left.0-200^{\circ} \mathrm{C}, 10 \mathrm{~K} \mathrm{~min}^{-1}\right)$ of ACMO_DAP-G.

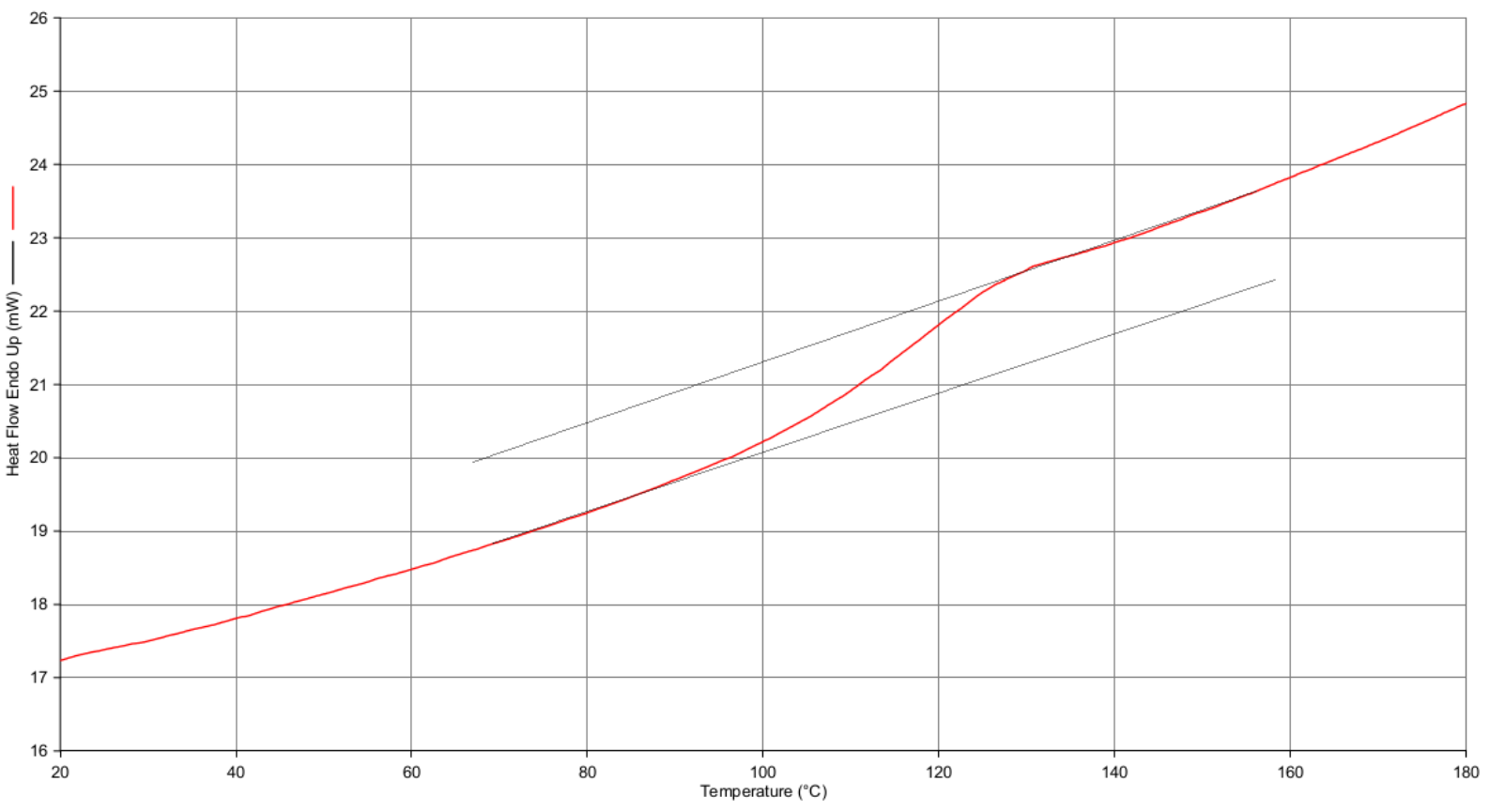

Figure S 32: DSC graph $\left(2^{\text {nd }}\right.$ heating, $\left.0-200^{\circ} \mathrm{C}, 10 \mathrm{~K} \mathrm{~min}^{-1}\right)$ of $A C M O \_X D A-G$. 


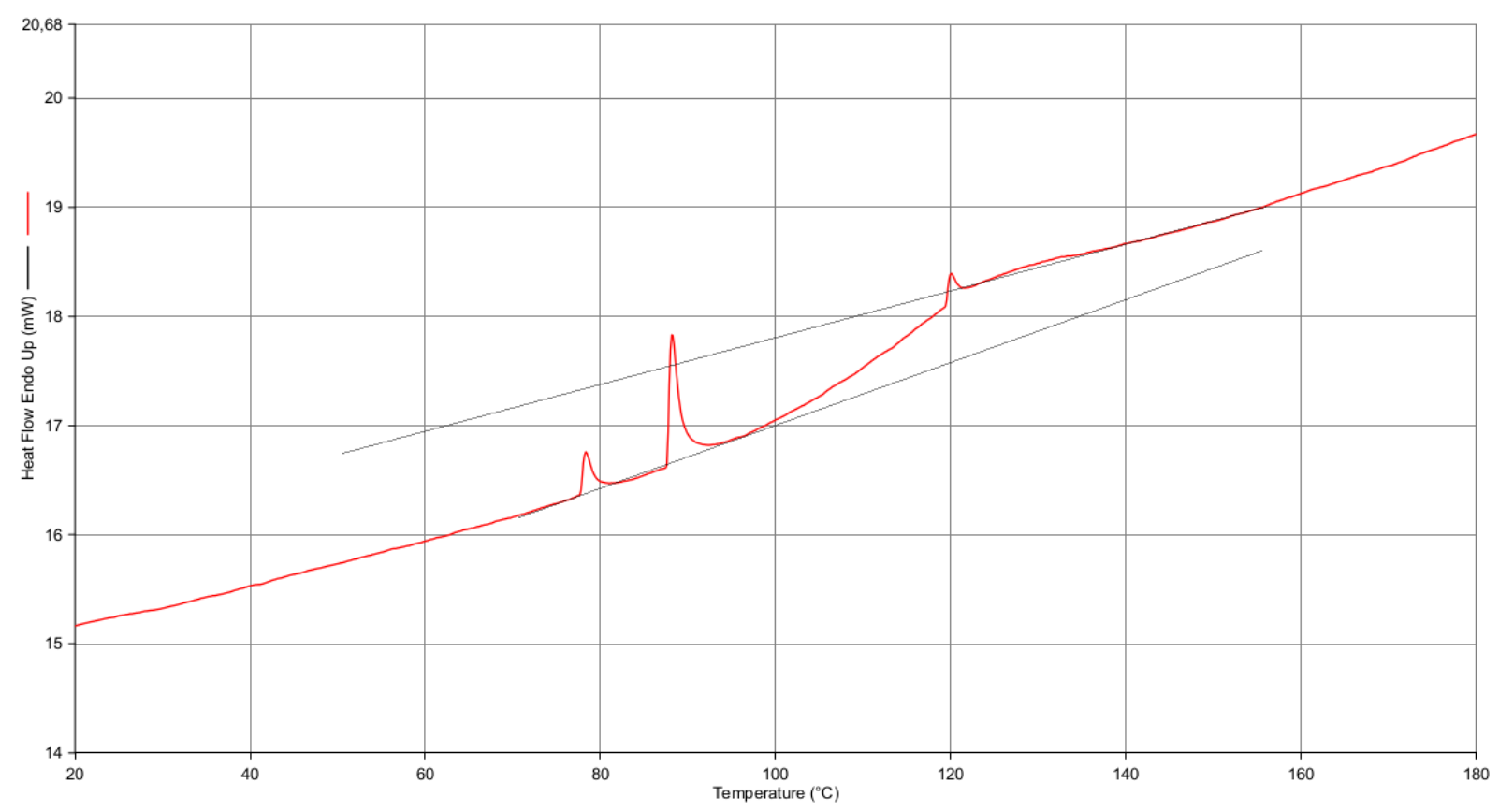

Figure S 33: DSC graph $\left(2^{\text {nd }}\right.$ heating, $\left.0-200^{\circ} \mathrm{C}, 10 \mathrm{~K} \mathrm{~min}^{-1}\right)$ of ACMO_CDMA-G.

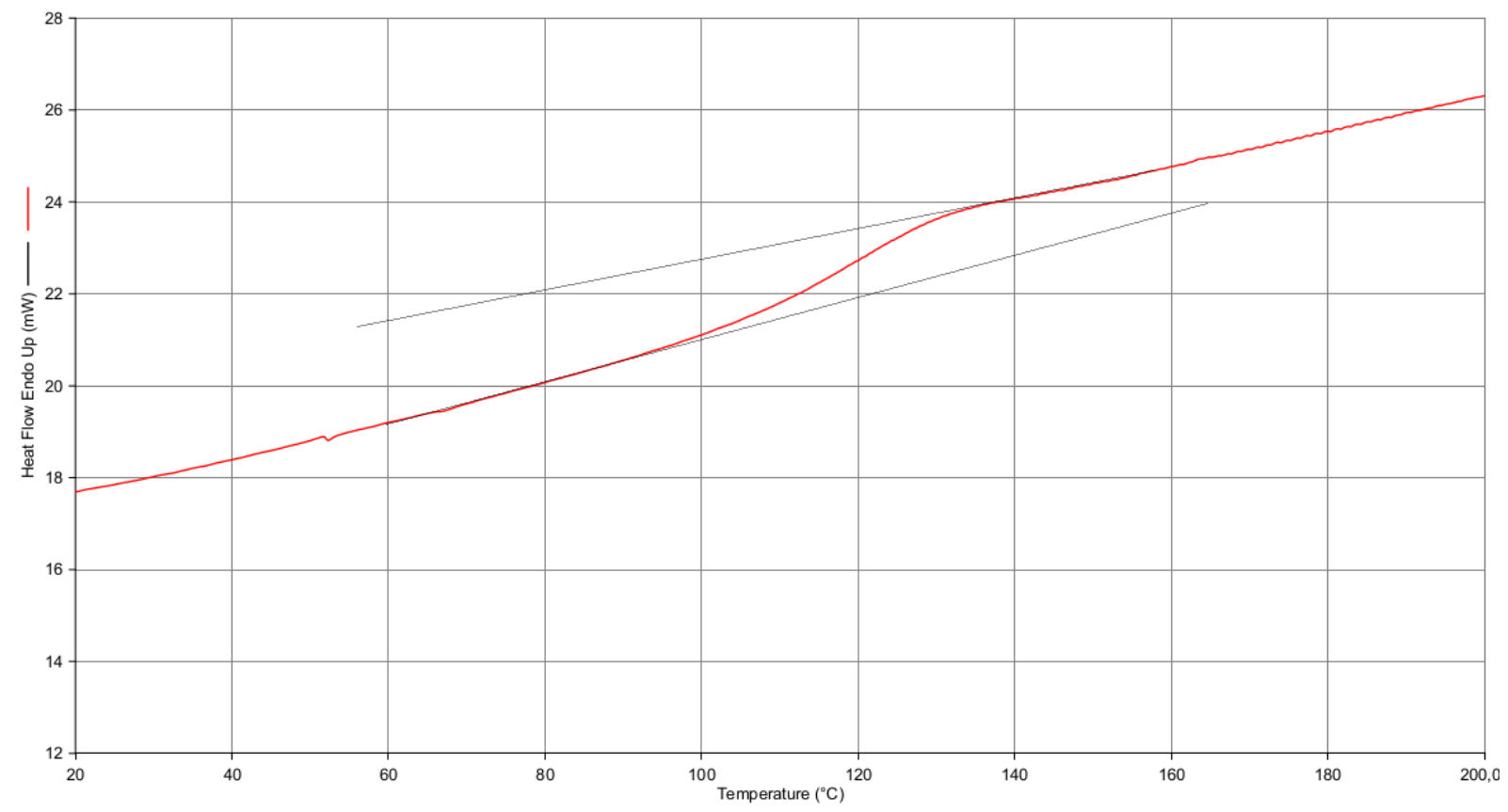

Figure S 34: DSC graph $\left(2^{\text {nd }}\right.$ heating, $\left.0-200^{\circ} \mathrm{C}, 10 \mathrm{~K} \mathrm{~min}^{-1}\right)$ of ACMO_IPDA-G. 


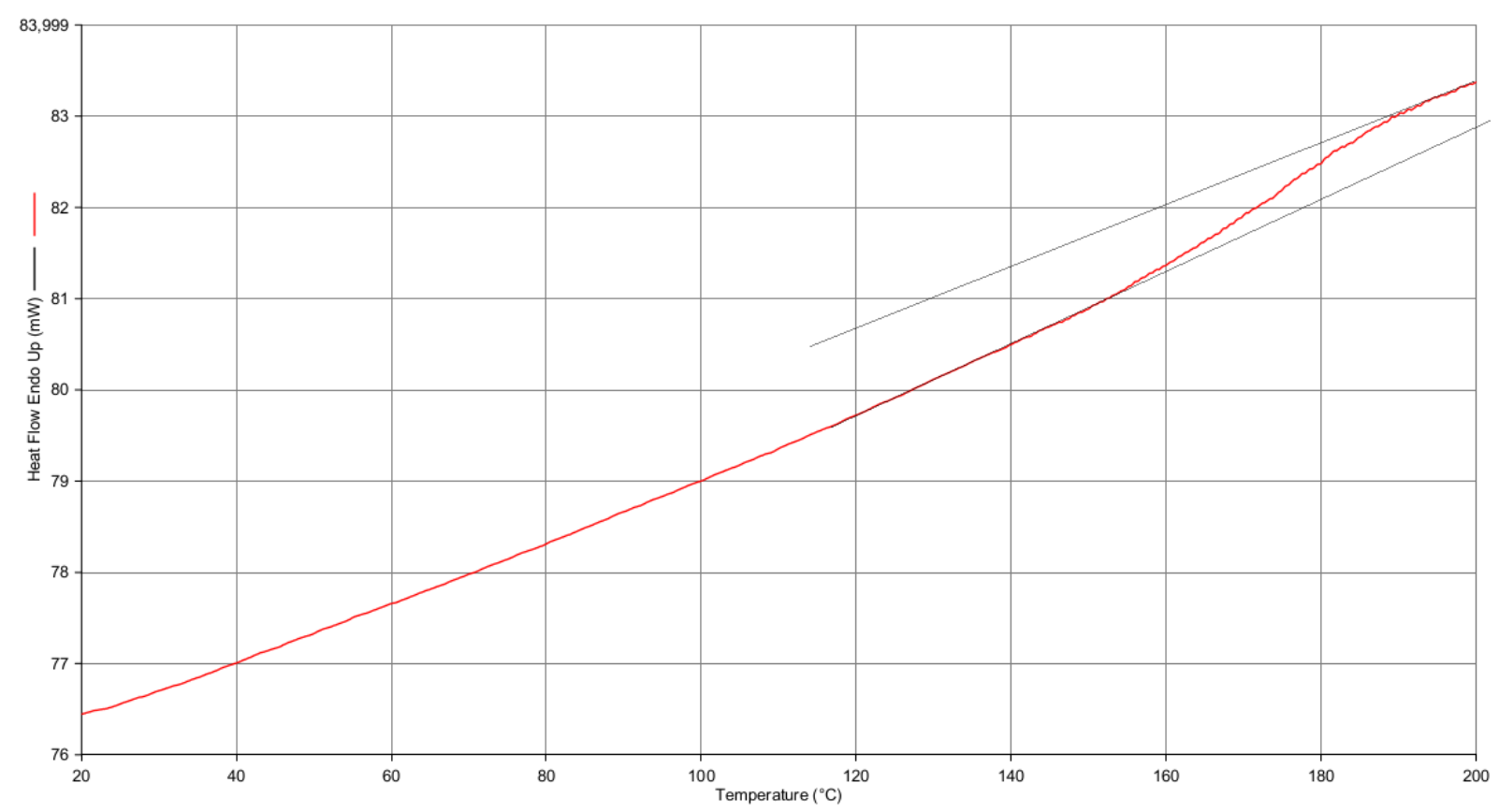

Figure S 35: DSC graph $\left(2^{\text {nd }}\right.$ heating, $\left.0-200^{\circ} \mathrm{C}, 10 \mathrm{~K} \mathrm{~min}^{-1}\right)$ of ACMO_DODA12 G5.

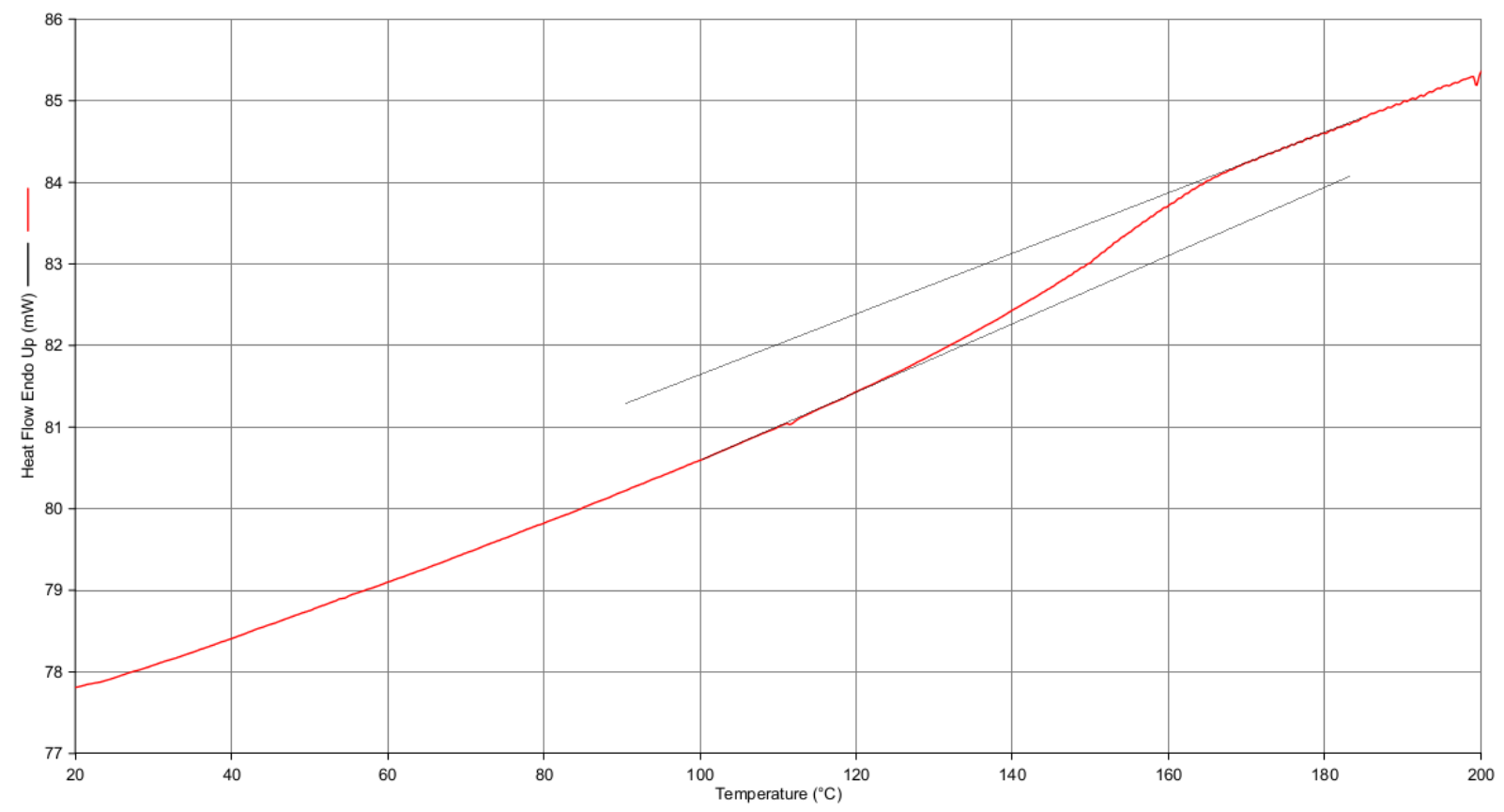

Figure S 36: DSC graph $\left(2^{\text {nd }}\right.$ heating, $\left.0-200^{\circ} \mathrm{C}, 10 \mathrm{~K} \mathrm{~min}^{-1}\right)$ of ACMO_DODA12 G5/GDMA. 
III) Tensile testing

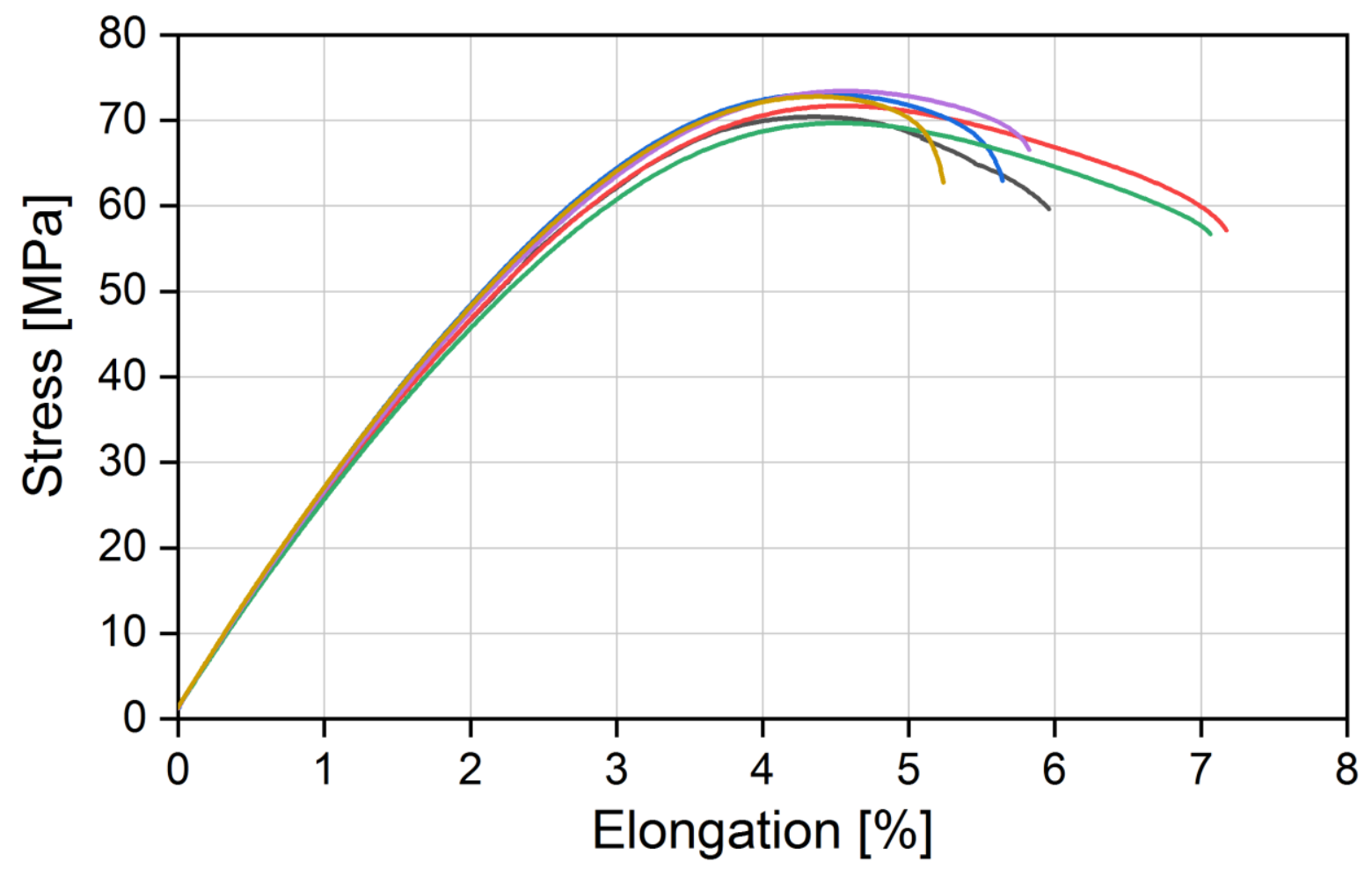

Figure S 37: Tensile testing (ISO 527-1/2_5A, $50 \mathrm{~mm} \mathrm{~min}^{-1}$ ) results for ACMO_Laromer.

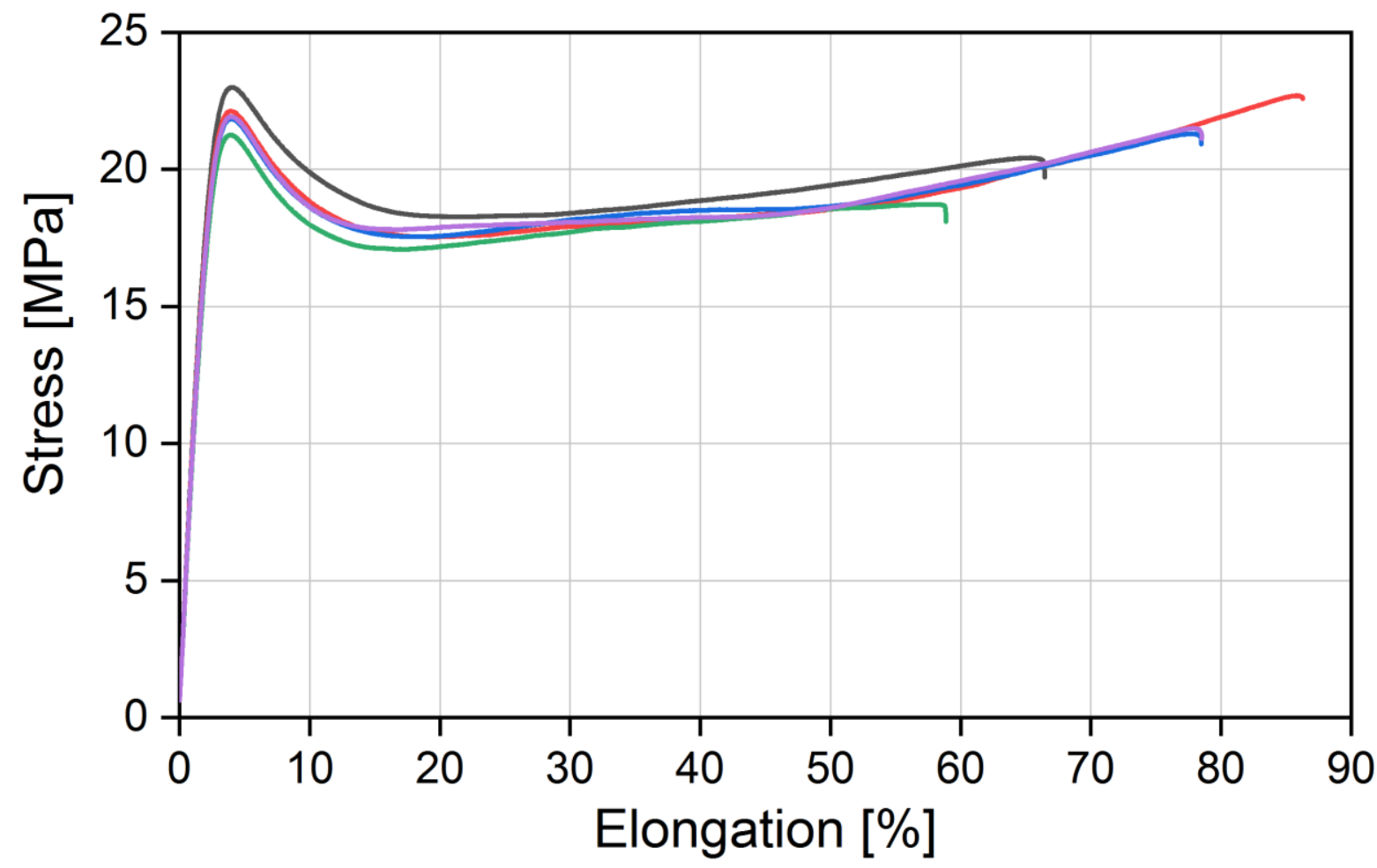

Figure S 38: Tensile testing (ISO 527-1/2_5A, $50 \mathrm{~mm} \mathrm{~min}^{-1}$ ) results for ACMO_PTHFA-G. 


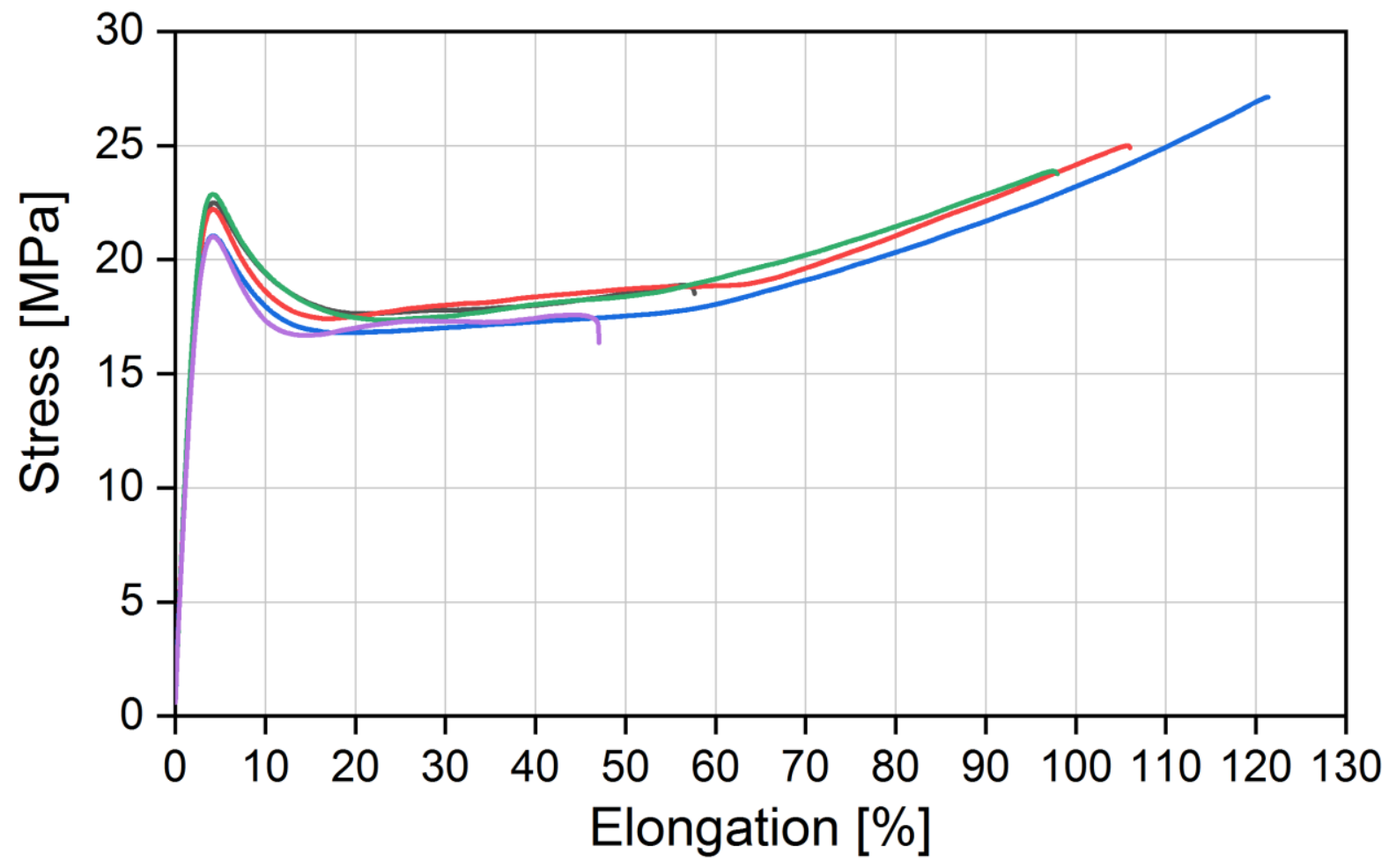

Figure S 39: Tensile testing (ISO 527-1/2_5A, $50 \mathrm{~mm} \mathrm{~min}^{-1}$ ) results for ACMO_JT3000-G.

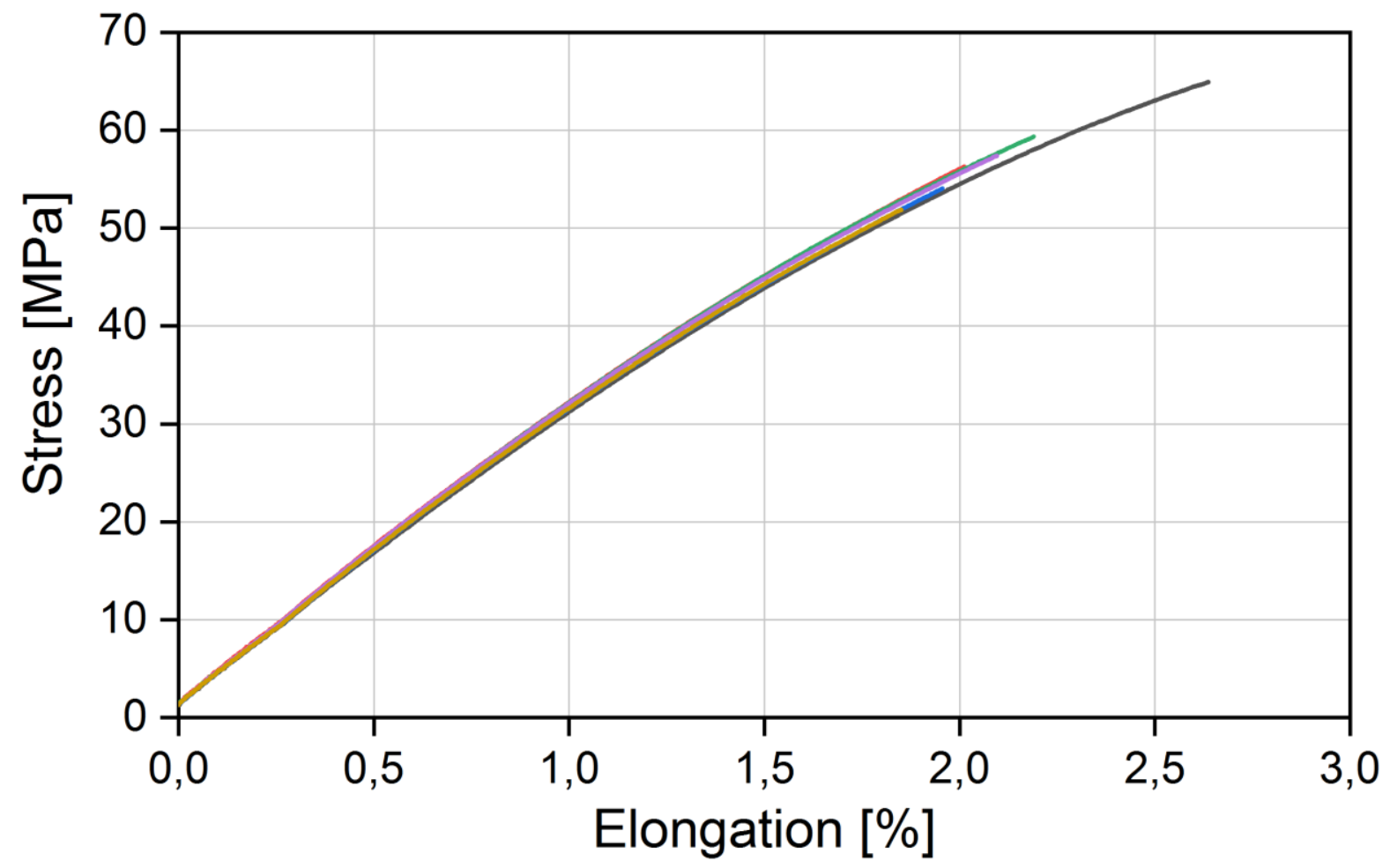

Figure S 40: Tensile testing (ISO 527-1/2_5A, $50 \mathrm{~mm} \mathrm{~min}^{-1}$ ) results for ACMO_JT403-G. 


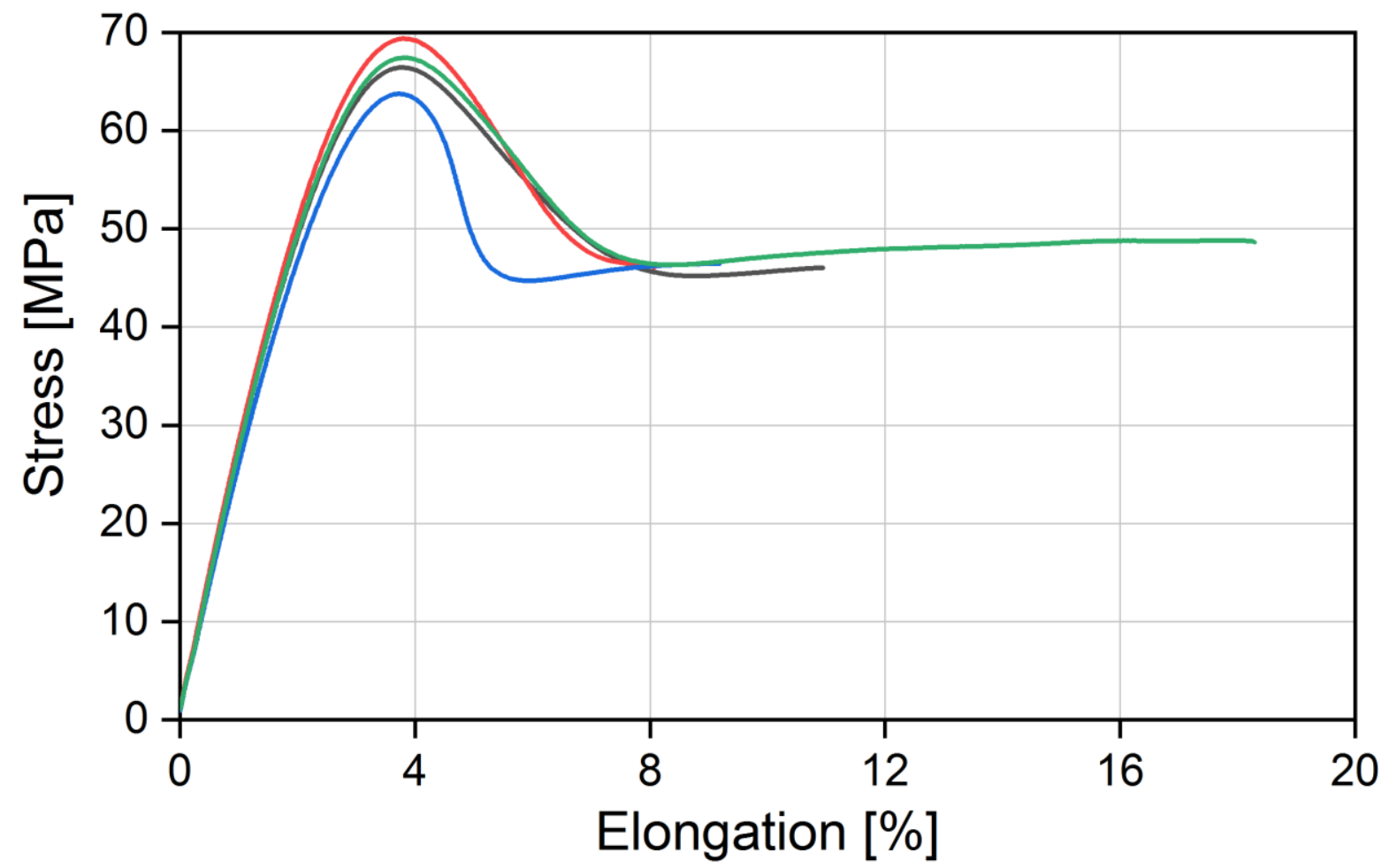

Figure S 41: Tensile testing (ISO 527-1/2_5A, $50 \mathrm{~mm} \mathrm{~min}^{-1}$ ) results for ACMO_TODA-G.

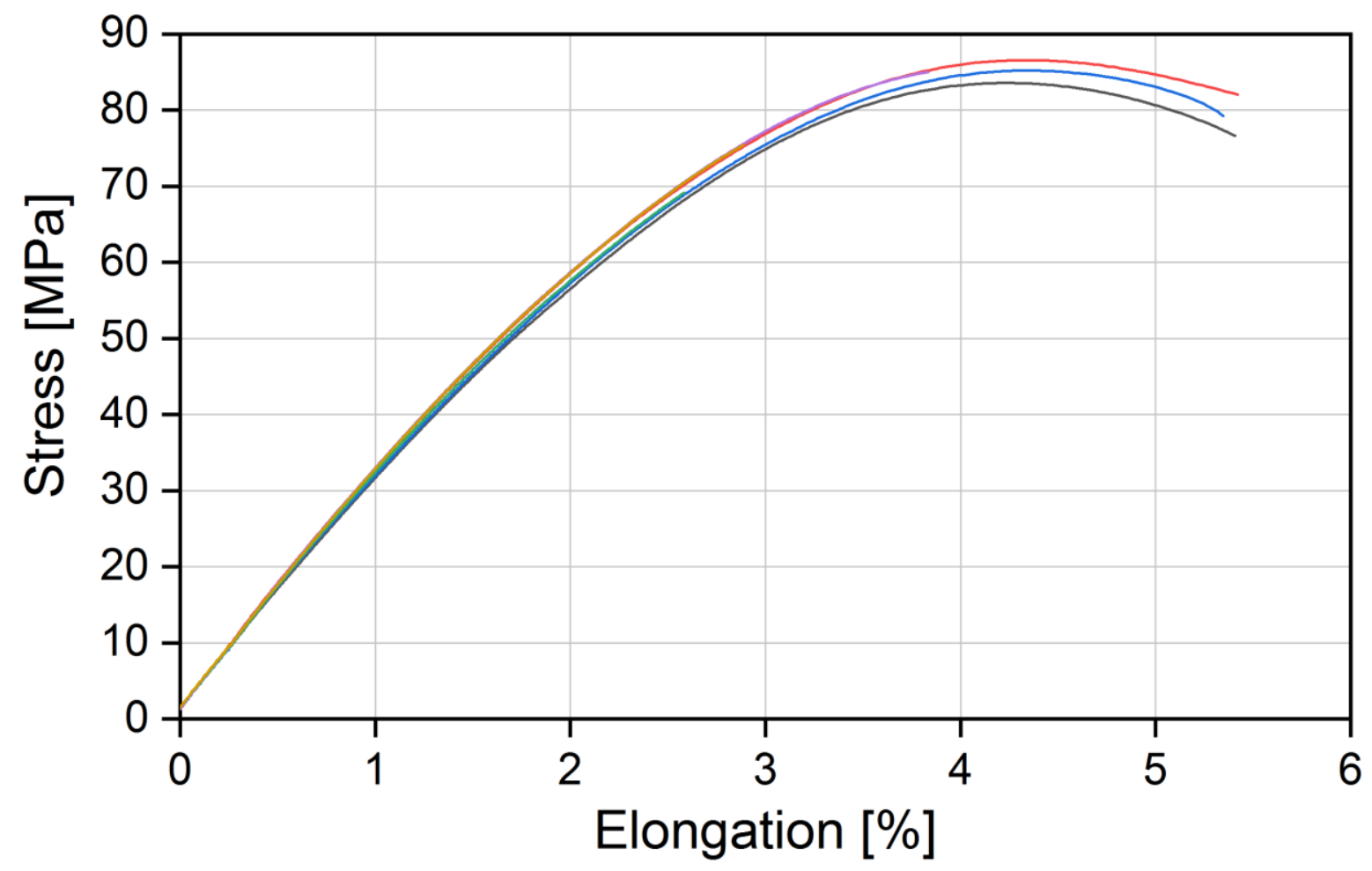

Figure S 42: Tensile testing (ISO 527-1/2_5A, $50 \mathrm{~mm} \mathrm{~min}^{-1}$ ) results for ACMO_DODA12 G. 


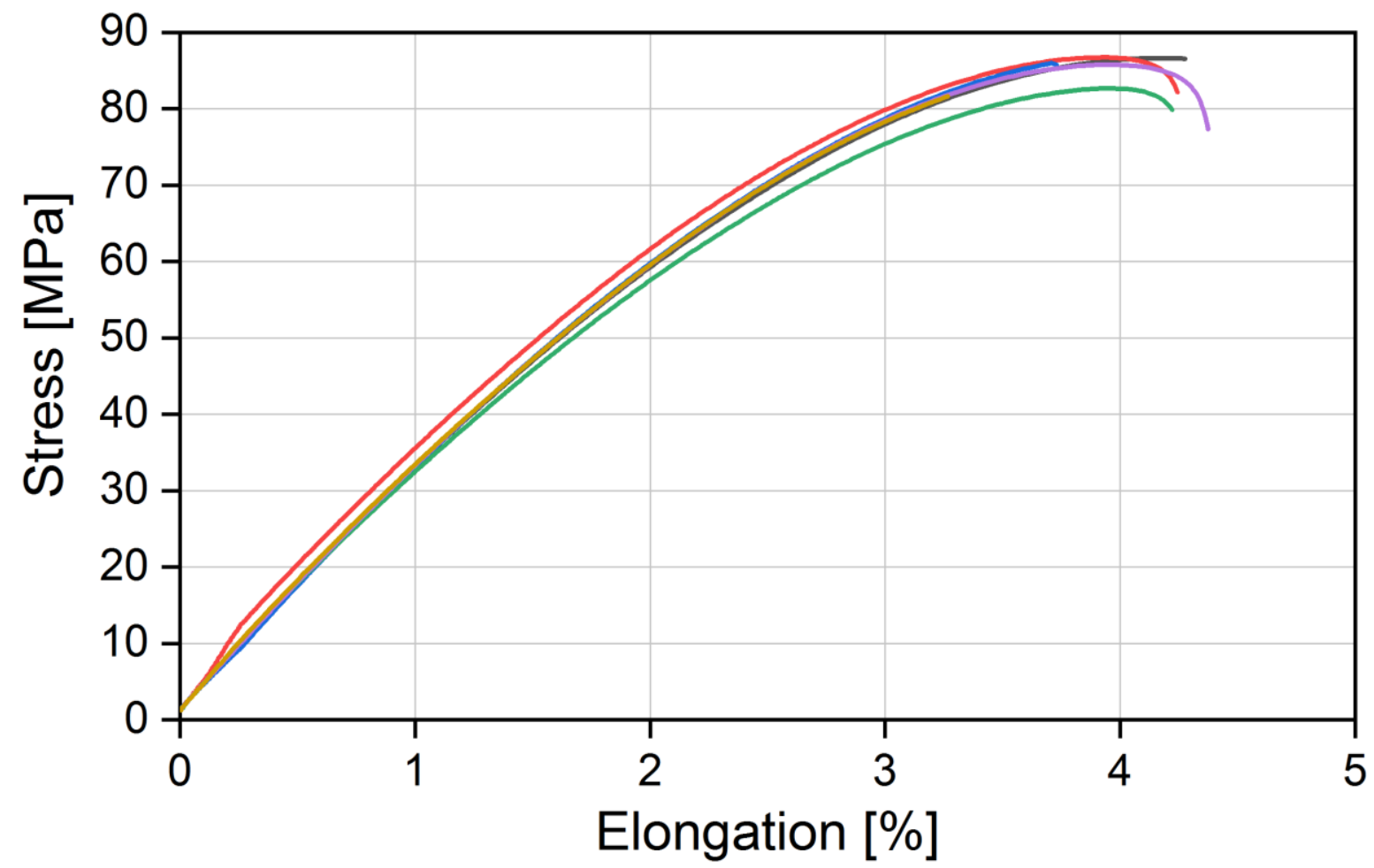

Figure S 43: Tensile testing (ISO 527-1/2_5A, $50 \mathrm{~mm} \mathrm{~min}^{-1}$ ) results for ACMO_DODA8-G.

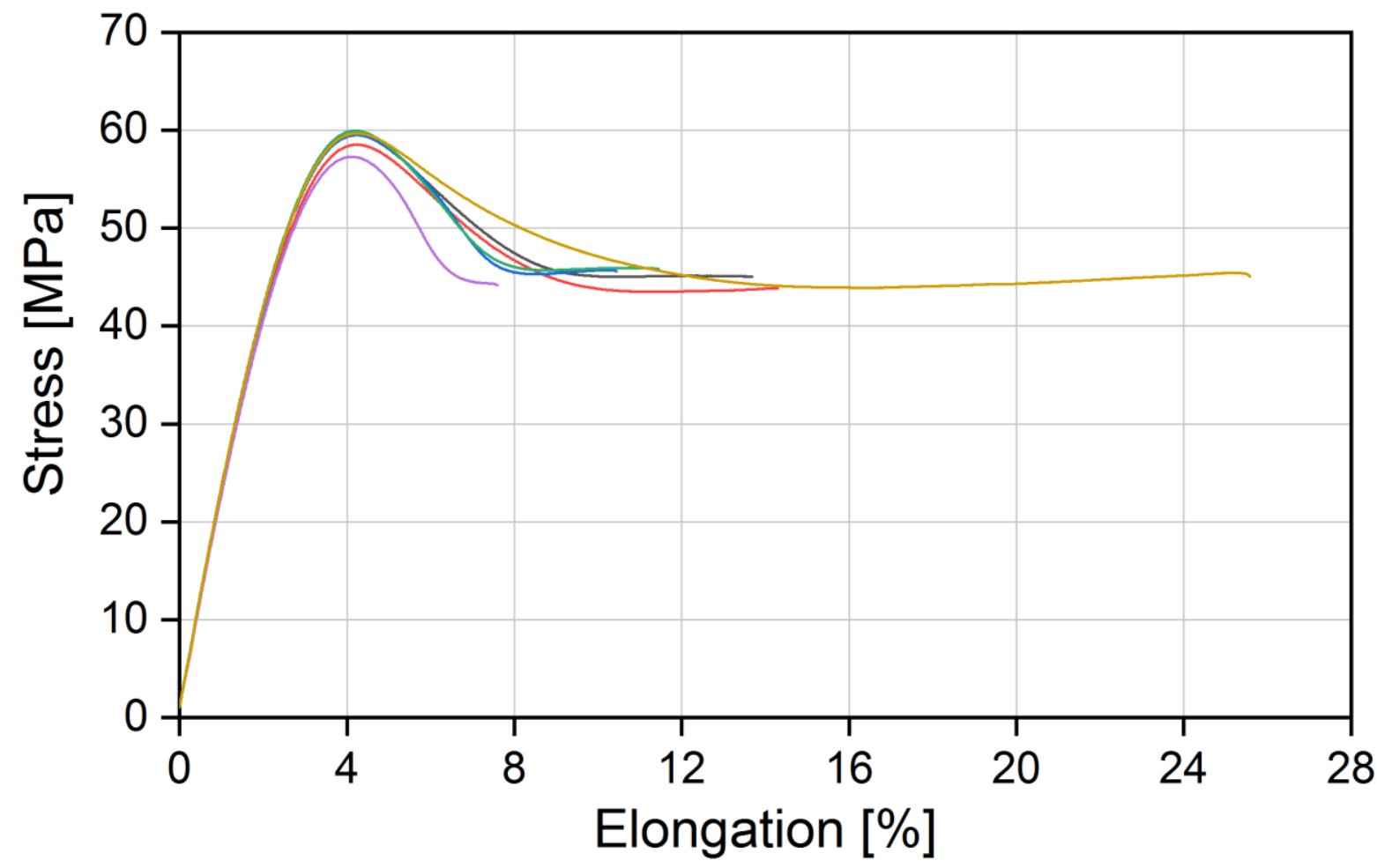

Figure S 44: Tensile testing (ISO 527-1/2_5A, $50 \mathrm{~mm} \mathrm{~min}^{-1}$ ) results for ACMO_PA-G. 


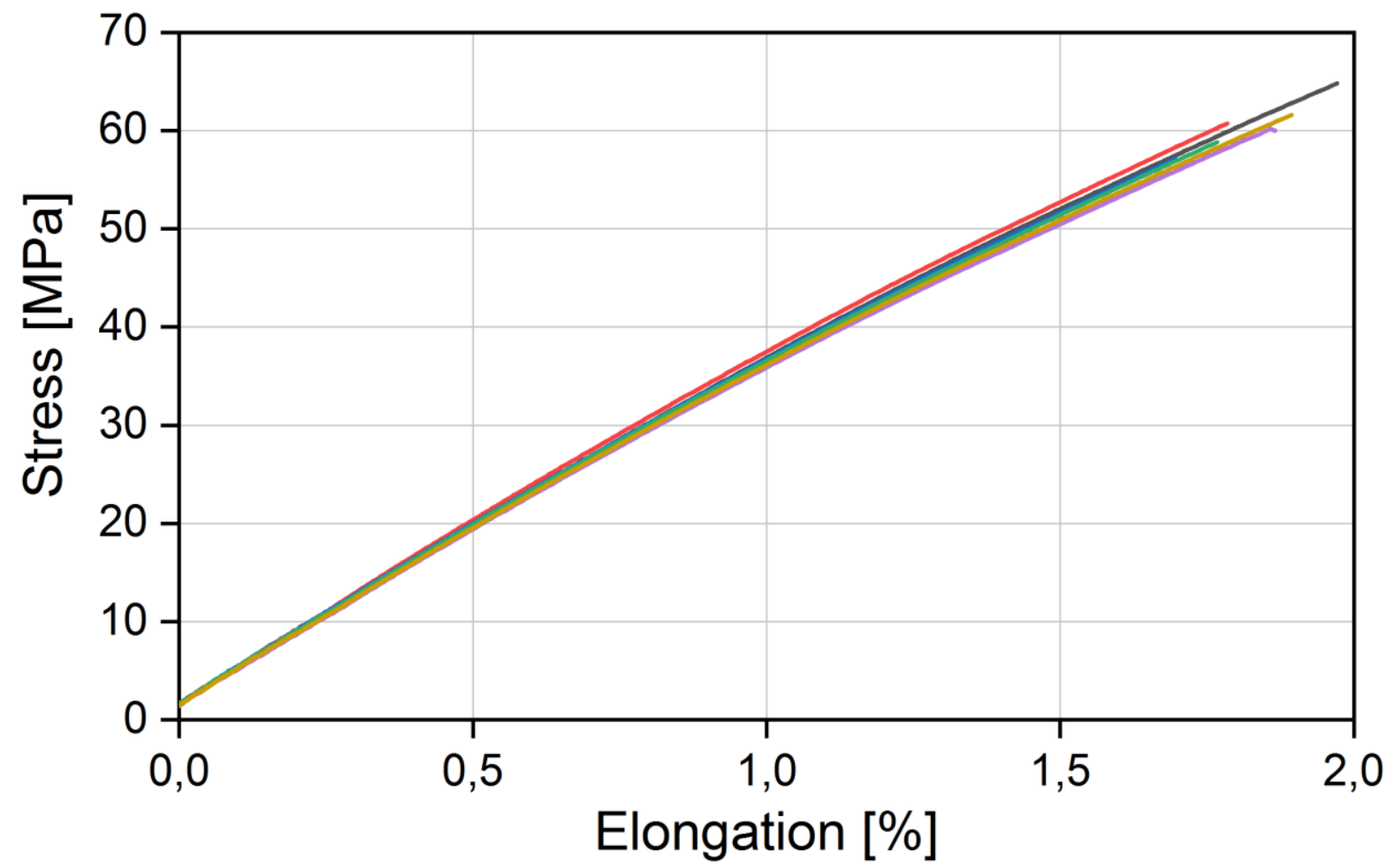

Figure S 45: Tensile testing (ISO 527-1/2_5A, $50 \mathrm{~mm} \mathrm{~min}^{-1}$ ) results for ACMO_DAP-G.

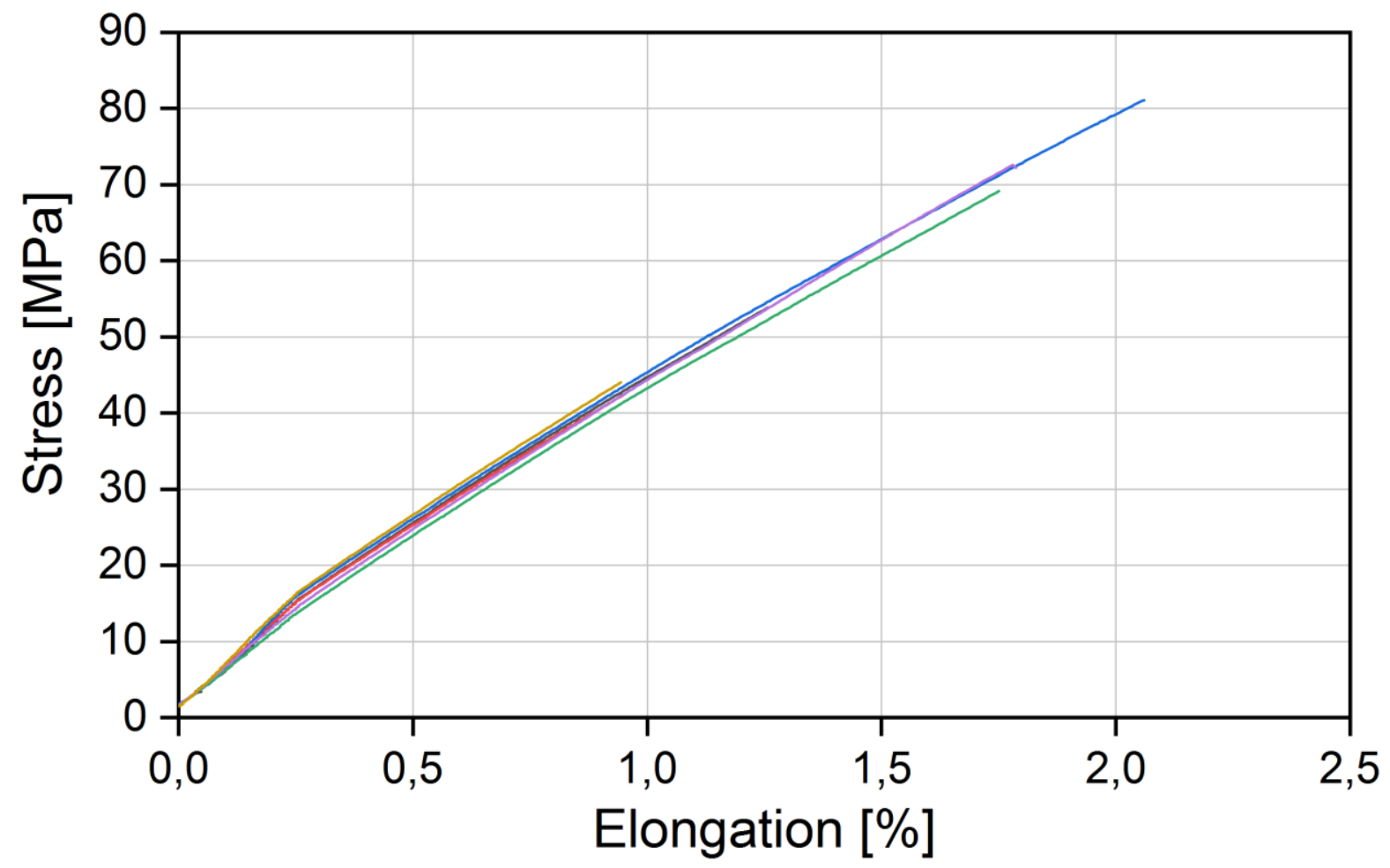

Figure S 46: Tensile testing (ISO 527-1/2_5A, $50 \mathrm{~mm} \mathrm{~min}^{-1}$ ) results for ACMO_XDA-G. 


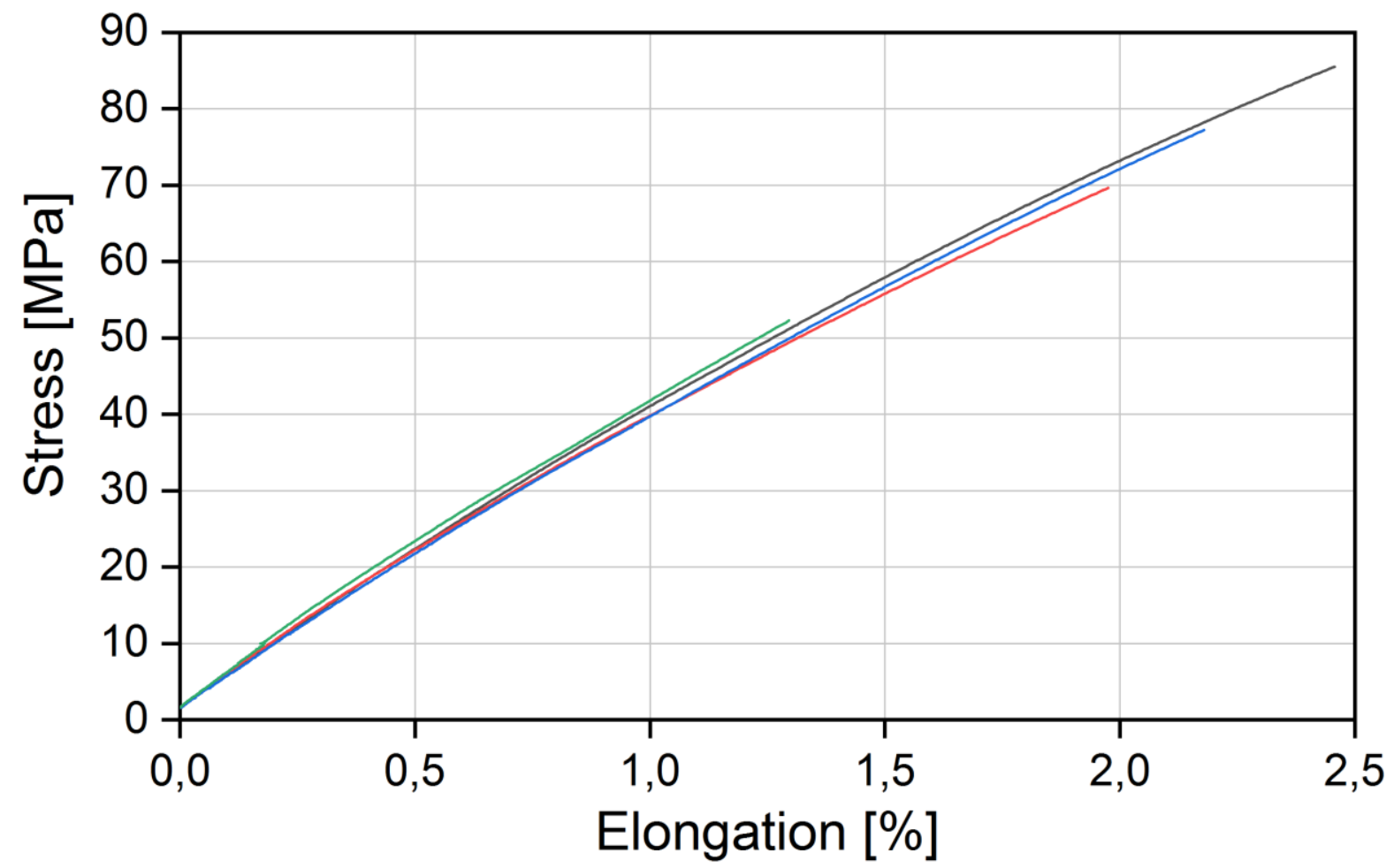

Figure S 47: Tensile testing (ISO 527-1/2_5A, $50 \mathrm{~mm} \mathrm{~min}^{-1}$ ) results for ACMO_CDMA-G.

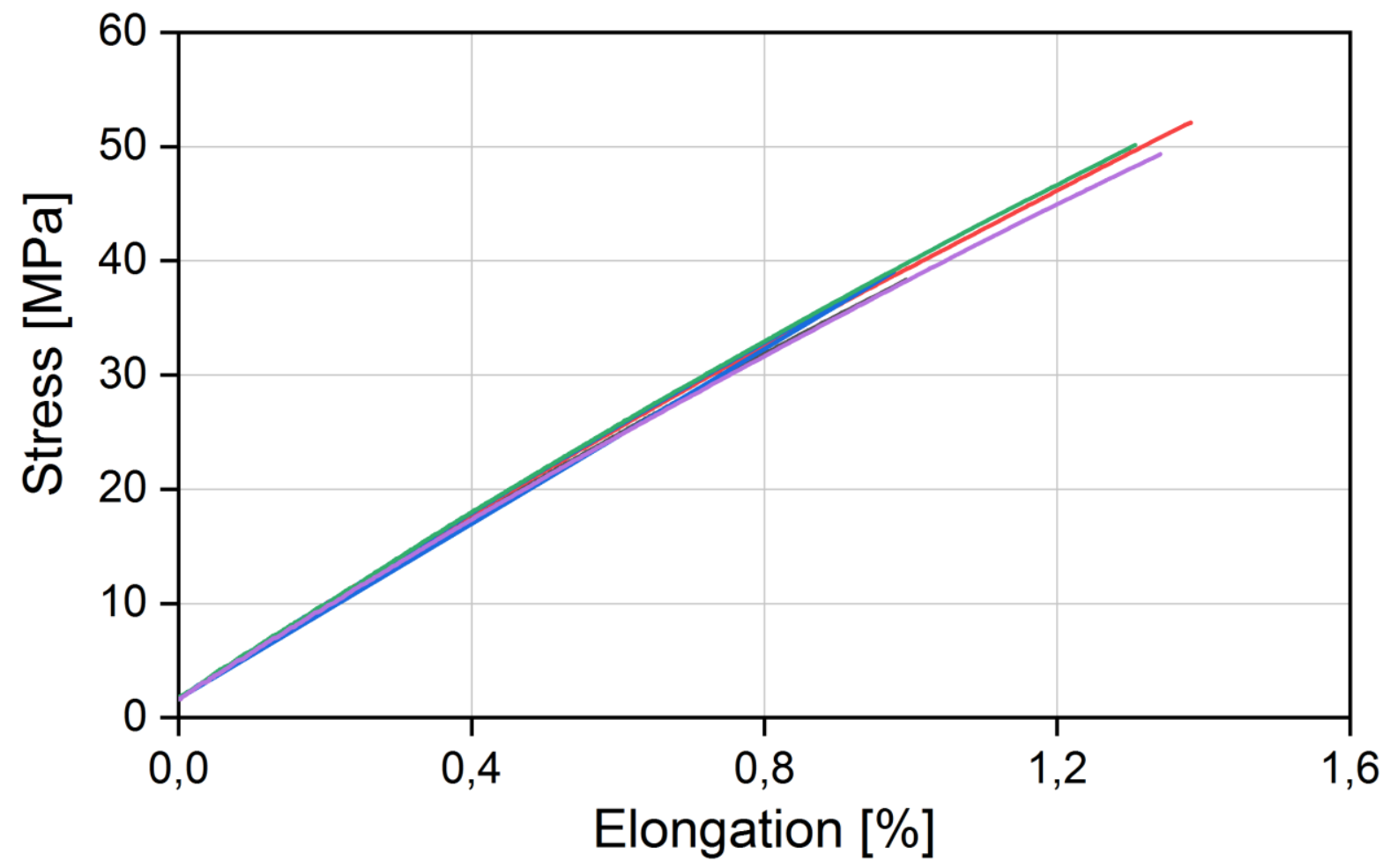

Figure S 48: Tensile testing (ISO 527-1/2_5A, $50 \mathrm{~mm} \mathrm{~min}^{-1}$ ) results for ACMO_IPDA-G. 


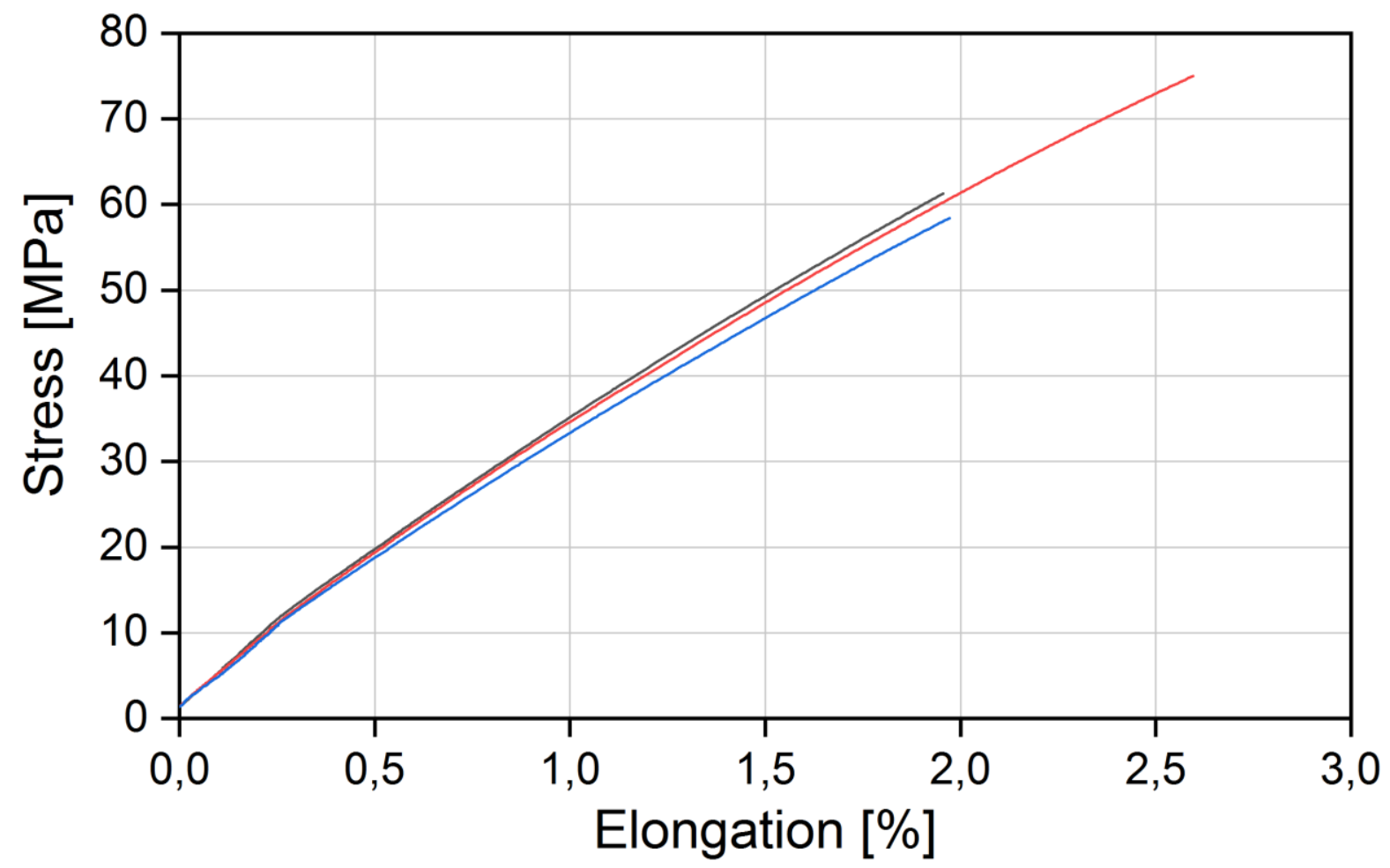

Figure S 49: Tensile testing (ISO 527-1/2_5A, $50 \mathrm{~mm} \mathrm{~min}^{-1}$ ) results for ACMO_DODA12 G5.

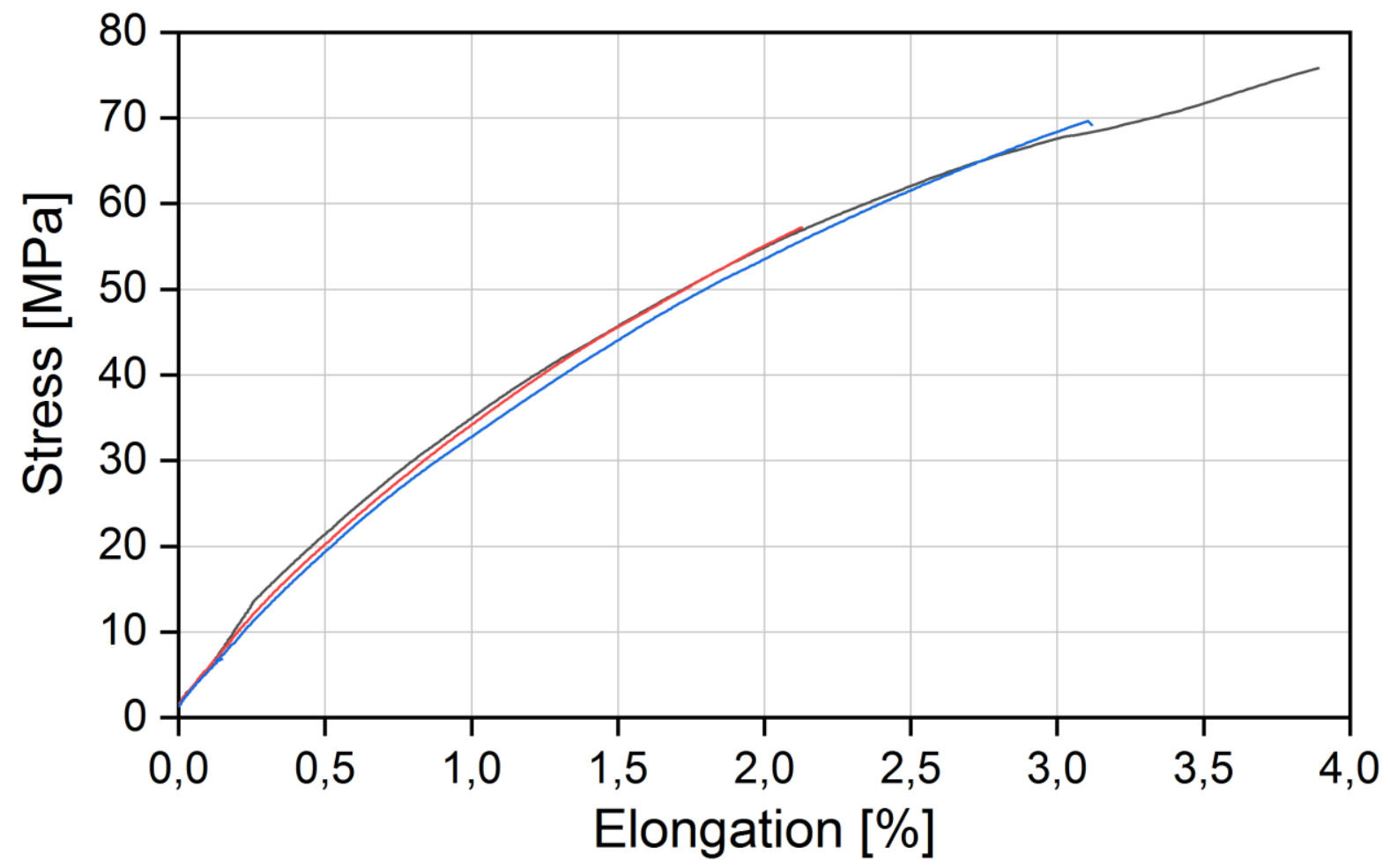

Figure S 50: Tensile testing (ISO 527-1/2_5A, $50 \mathrm{~mm} \mathrm{~min}^{-1}$ ) results for ACMO_DODA12 G5/GDMA. 


\section{IV) Rheological experiments}

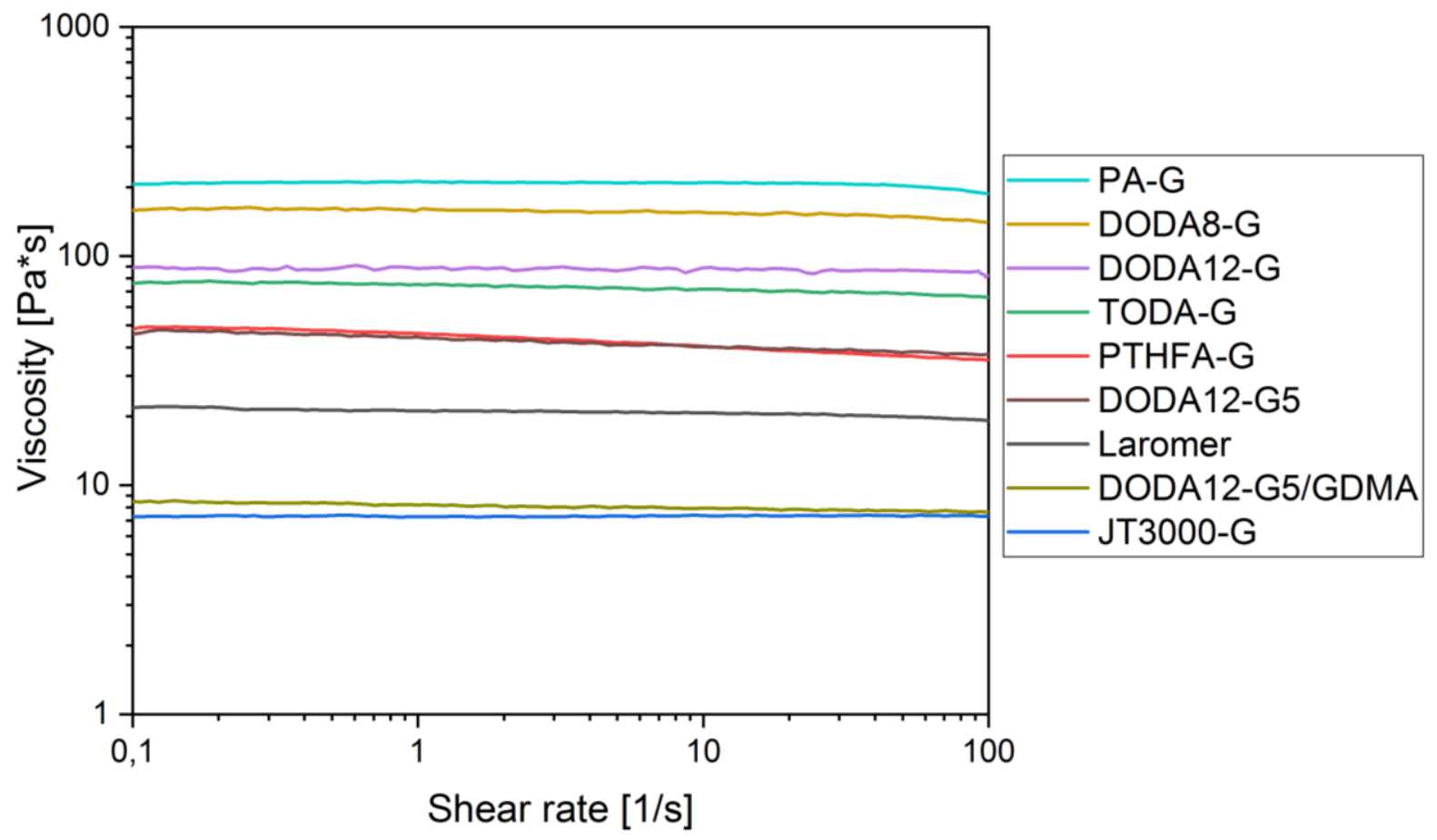

Figure S 51: Measured viscosity in dependence from the shear rate for synthesized methacrylate building blocks (plateplate rheometer, from 0.1 to $100 \mathrm{~s}^{-1}, 100$ steps, logarithmic, $5 \mathrm{~s}$ per step, $3 \mathrm{~s}$ integration time).

\section{V) Calculation of the degree of oligomerization}<smiles>C=C(C)C(=O)OCC(O)COC(=O)NCCCCCNC(=O)OCC(O)COC(=O)C(C)C(C)NCCCCCNC(=O)OCC(O)COC(=O)C(=C)C</smiles>

Eq. 1 follows from the weight portion ratio of GCMA and the diamine.

$\frac{n_{A}}{n_{G}}=0.6$

Eq. 2 describes the ratio of the GCMA and the Diamine building blocks within the oligomer and assumes full cyclic carbonate conversion.

$\frac{n_{A}}{n_{G}}=\frac{m+1}{m+2}$

Combining eq. 1 and eq. 2 allows to solve after the oligomerization parameter m:

$0.6=\frac{m+1}{m+2} \quad \rightarrow \quad m=0.5$

(eq. S3) 


\section{VI) Pictures}

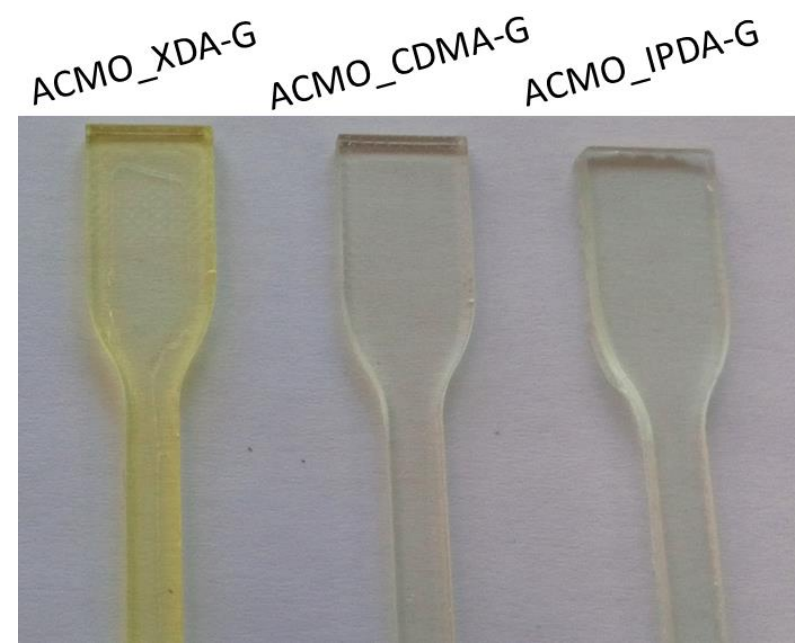

Figure S 52: Photograph of cured tensile testing specimen.

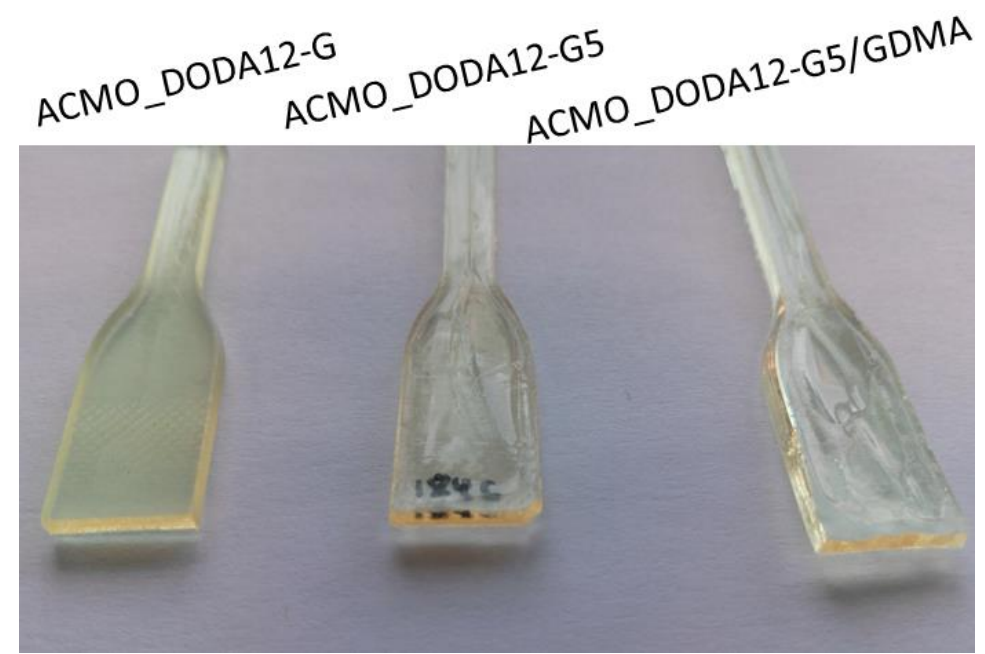

Figure S 53: Photograph of cured tensile testing specimen. 